Research Article

\title{
Analysis of Vibration Control of Nonlinear Beam Using a Time-Delayed PPF Controller
}

\author{
Yueli Chen $\mathbb{1}^{1}$ and Juhong $\mathbf{G e}^{2}$ \\ ${ }^{1}$ Henan Academy of Big Data, Zhengzhou University, Zhengzhou 450001, China \\ ${ }^{2}$ School of Mathematics and Information Science, Henan University of Economics and Law, Zhengzhou 450046, China \\ Correspondence should be addressed to Yueli Chen; chenyueli@zzu.edu.cn
}

Received 5 April 2020; Revised 13 July 2020; Accepted 19 July 2020; Published 6 August 2020

Academic Editor: Matteo Strozzi

Copyright ( $) 2020$ Yueli Chen and Juhong Ge. This is an open access article distributed under the Creative Commons Attribution License, which permits unrestricted use, distribution, and reproduction in any medium, provided the original work is properly cited.

\begin{abstract}
This paper presents a study on the performance of a positive position feedback (PPF) controller to suppress the vibration of a horizontal beam under vertical excitation. Time delays in the control loop are taken into consideration to study their effects on the controller performance and the stable region. The integral iterative method is conducted to obtain a second-order approximate solution and the corresponding amplitude equations for the considered system. The stability of the steady-state solutions is ascertained using a combination of Floquet theory and Hill's determinant. The maximum limits of time delays at which the system remains stable have been determined for different values of control parameters. And the effects of various control parameters on the existence of multiple-solution region are investigated. The analysis illustrates that the appearance of time delay and the elimination of controller damping coefficient are the two main factors to enhance the nonlinear characteristics of the controlled system. The points at which the steady-state amplitude of the main system reaches its minimum are studied analytically. The analyses show that the analytical results are in excellent agreement with the numerical simulations.
\end{abstract}

\section{Introduction}

Active vibration control has been used to suppress the undesired vibrations in different systems for many years. Various types of controllers are designed to channel the excess energy from the excited systems to the slave ones. Applications of the positive position feedback controller to reduce a system's vibrations have been intensively developed in recent years. Shan et al. [1] employed a positive position feedback controller to suppress multi-mode vibrations while slewing the single-link flexible manipulator. Experimental results showed that PPF controller is more effective than the L-type velocity feedback controller while the slewing process was realized. Jun [2] employed an active linear absorber based on PPF control to reduce the high-amplitude vibration of the single-mode of the flexible beam when subjected to primary resonance excitation. It showed that the control scheme possessed a wide suppression bandwidth if the absorber's frequency was properly tuned. Numerical and experimental researches on four types of controllers including PPF controller applied to nonlinear beam models were presented in [3]. The results showed that PPF and NSC controllers were most effective in suppressing the highamplitude vibration of flexible composite beam structure. Mitura et al. [4] presented numerical results for the PPF control method applied to strongly nonlinear horizontal and vertical beam models. They demonstrated that the PPF controller was more effective in reducing the vibration of the vertical beam than that of the horizontal beam. El-Ganaini et al. [5] presented an analytical study for positive position feedback controller to suppress the vibration amplitude of a nonlinear dynamical model when the primary resonance and the 1:1 internal resonance occurred simultaneously. They found that it was necessary to tune the controller's natural frequency to the same value of the excitation frequency in the control process. In [6], a new nonlinear modified PPF controller was introduced to suppress the nonlinear vibration at primary resonance. It was 
demonstrated that the nonlinear modified PPF controller provided a higher level of suppression in the overall frequency domain compared with the conventional PPF controller. El-Sayed and Bauomy [7] employed two PPF controllers to reduce the vertical vibration in the vertical conveyors. It was shown that PPF controllers were very suitable for small natural frequency dynamical systems subjected to primary resonance excitations. Kandil and Eissa [8] overcame the drawback of the PPF controller by coupling additional NSCs to the main system to impose a V-curve at each one of the peaks out of the effective frequency bandwidth. In this control process, the two peaks could be suppressed to acceptable levels. Saeed and Kamel [9] applied a tuned PPF controller to suppress the lateral vibrations of a Jeffcott-rotor system. They concluded that the controller could reduce the vibration amplitudes close to zero at any spinning speed even at large disc eccentricity. In [10], a positive position feedback controller was proposed to suppress the nonlinear vibrations of a horizontally supported Jeffcott-rotor system. They found that the nonlinear PPF controller could eliminate the nonlinear phenomena of the Jeffcott-rotor system.

Time delays inherently exist in many active control systems. They may induce complex dynamic behavior such as undesirable bifurcations, quasiperiodic motions, and chaotic behavior, etc. They limit the performance of active control. Therefore, it is necessary to investigate the effect of time delays on the active control process. In [11-13], Saeed et al. proposed three different controllers to suppress the vibrations of nonlinear Jeffcott-rotor systems. They presented the regions at which the system solutions were stable on the $\tau_{1}-\tau_{2}$ plane. The methods for selecting the optimal values of time delays were proposed. They found that time delays could not only improve the vibration suppression performance, but also increase the vibration amplitudes and destabilize the controlled system. In [14], a nonlinear time delay saturation-based controller was proposed to suppress the vibrations of a nonlinear beam. They proposed a concept of "vibration suppression region" at which the amplitudedelay's response curves exhibit stable solution. They concluded that time delays could adjust the effective frequency bandwidth of the saturation controller and avoid the occurrence of the controller overload. El-Ganaini et al. [15] proposed a time-delayed PPF controller to suppress the horizontal vibration of a magnetically levitated body subjected to multiple force excitations. They concluded that the amplitudes of the controlled system did not depend only on a certain delay, but on the sum of two delays. Similar results also appeared in [16]. The authors demonstrated that time margins which indicated the safe region of operation depended on the overall delay of the controlled system. Kandil and El-Ganaini [17] utilized a time-delayed PPF controller to reduce the nonlinear oscillations of the compressor blade system subjected to a primary excitation at $1: 1$ internal resonance. They investigated the effect of time delay on the control of rotating blade vibrations and presented the safe region of operation.

In this paper, a positive position feedback controller is utilized to suppress the vibration of a nonlinear horizontal beam under vertical excitation. Time delays in the control loop are taken into consideration in this work. We get an approximate solution by applying the integral iterative method. The stability of the system is investigated by applying a combination of Floquet theory and Hill's determinant. The safe operation region of time delays is investigated for different values of control parameters. The main factors affecting the nonlinear characteristics of the controlled system are analyzed. In addition, the points at which the steady-state amplitude of the main system reaches its minimum are investigated analytically. Numerical simulations are presented to validate the analytical predictions. Finally, a comparison with the previously published works is included in the end of this paper.

\section{Mathematical Model}

The nonlinear differential equation that describes the vibration of a nonlinear horizontal beam under vertical excitation [4] is given as follows:

$$
\ddot{v}+2 \mu \omega_{1} \dot{v}+\omega_{1}^{2} v+\beta v^{3}+\delta\left(v \dot{v}^{2}+v^{2} \ddot{v}\right)=\xi y_{0} \Omega^{2} \sin (\Omega t) .
$$

The model of the horizontal beam and the experimental setup were presented in [16]. By integrating a time-delayed PPF controller to the nonlinear beam, the equation governing the dynamics of the controlled system is suggested as

$$
\begin{aligned}
& \ddot{v}+2 \mu \omega_{1} \dot{v}+\omega_{1}^{2} v+\beta v^{3}+\delta\left(v \dot{v}^{2}+v^{2} \ddot{v}\right) \\
& =\xi y_{0} \Omega^{2} \sin (\Omega t)+\gamma u\left(t-\tau_{1}\right) \\
& \ddot{u}+2 \zeta \omega_{2} \dot{u}+\omega_{2}^{2} u=\alpha v\left(t-\tau_{2}\right)
\end{aligned}
$$

where $v$ denotes the response of the main system (the horizontal beam), $u$ denotes the response of the PPF controller, $\omega_{1}$ is the natural frequency of the main system, $\mu$ is the damping ratio of the main system, $\beta$ is the curvature nonlinearity coefficient, $\delta$ denotes the inertia nonlinearity coefficient, $\zeta$ is the damping ratio of the controller, $\omega_{2}$ is the natural frequency of the controller, $y_{0}$ and $\Omega$ represent the amplitude and frequency of the support motion, $\gamma$ denotes the control signal gain, $\alpha$ denotes the feedback signal gain, and $\tau_{1}, \tau_{2}$ are time delays.

\section{Analytical Solutions}

In [18], we improved the integral equation method introduced by Schmidt and Tondl [19] by adding time delay terms and rewriting the successive program. We renamed the improved method as the integral iterative method because of more iterative processes. Although these two methods are less known than other methods, they still have many advantages. In [19], the authors showed that the use of small parameters could lead to solutions of every degree of accuracy. The mechanism of the two methods is simple and clear. They are easier to program than many other perturbation methods. In our previous work [20], we found that the accuracy of the integral iterative method is much better than that of the multiple scales method when dealing 
with the single-degree-of-freedom problem. In this section, the integral iterative method is applied to obtain the second-order approximations and the amplitude equations for the system response. A more detailed introduction of the integral iterative method is given in Appendix.

From the previously published works $[4,5,16,21]$, it is concluded that the simultaneous resonance occurs when $\Omega=\omega_{1}$ and $\omega_{1}=\omega_{2}$. To study the PPF control, two detuning parameters $\sigma_{1}$ and $\sigma_{2}$ are introduced as follows:

$$
\begin{gathered}
\Omega=\omega_{1}+\sigma_{1}, \\
\omega_{2}=\omega_{1}+\sigma_{2} .
\end{gathered}
$$

We introduce a dimensionless time again by

$$
\begin{aligned}
& t_{1}=\Omega t, \\
& \tau_{1}^{*}=\Omega \tau_{1}, \\
& \tau_{2}^{*}=\Omega \tau_{2} .
\end{aligned}
$$

Here, for simplicity, we still replace $t_{1}, \tau_{1}^{*}, \tau_{2}^{*}$ by $t, \tau_{1}, \tau_{2}$. So, the closed loop system is transformed into the following form:

$$
\begin{aligned}
\ddot{v}+v= & \frac{1}{\Omega^{2}}\left[-2 \mu \omega_{1} \Omega \dot{v}-\left(\sigma_{1}^{2}-2 \Omega \sigma_{1}\right) v-\beta v^{3}-\delta \Omega^{2}\left(v \dot{v}^{2}\right.\right. \\
& \left.\left.+v^{2} \ddot{v}\right)+\xi y_{0} \Omega^{2} \sin t+\gamma u\left(t-\tau_{1}\right)\right], \\
\ddot{u}+u= & \frac{1}{\Omega^{2}}\left[-2 \zeta \omega_{2} \Omega \dot{u}+2 \Omega\left(\sigma_{1}-\sigma_{2}\right) u-\left(\sigma_{1}-\sigma_{2}\right)^{2} u\right. \\
& \left.+\alpha v\left(t-\tau_{2}\right)\right] .
\end{aligned}
$$

For equation (5), $\lambda_{i}=1, i=1,2$, the corresponding generalized Green's functions are

$$
\begin{aligned}
G_{i}[t, \sigma]= & \frac{1}{\pi}\left[\frac{1}{2}-\cos (t-\sigma)+\sum_{j=2}^{\infty} \frac{\cos j(t-\sigma)}{1-j^{2}}\right], \quad i=1,2, \\
G_{i}\left[t-\tau_{k}, \sigma\right]= & \frac{1}{\pi}\left[\frac{1}{2}-\cos \left(t-\tau_{k}-\sigma\right)\right. \\
& \left.+\sum_{j=2}^{\infty} \frac{\cos j\left(t-\tau_{k}-\sigma\right)}{1-j^{2}}\right], \quad i=1,2, k=1,2 .
\end{aligned}
$$

From equations (A.7), (A.8), and (A.9) in Appendix, we get the first approximation in the following form:

$$
\begin{aligned}
v_{1}(t) & =r_{1} \cos t+s_{1} \sin t, \\
u_{1}(t) & =r_{2} \cos t+s_{2} \sin t, \\
v_{1}\left(t-\tau_{k}\right) & =r_{1} \cos \left(t-\tau_{k}\right)+s_{1} \sin \left(t-\tau_{k}\right), \quad k=1,2, \\
u_{1}\left(t-\tau_{k}\right) & =r_{2} \cos \left(t-\tau_{k}\right)+s_{2} \sin \left(t-\tau_{k}\right), \quad k=1,2 .
\end{aligned}
$$

Substituting equations (7)-(10) into equations (A.7), (A.8), and (A.9), we get the second approximations in the following:

$$
\begin{aligned}
v_{2}(t)= & \frac{1}{\pi \Omega^{2}} \int_{0}^{2 \pi}\left(\frac{1}{2}-\cos (t-\sigma)+\sum_{j=2}^{\infty} \frac{\cos j(t-\sigma)}{1-j^{2}}\right) \\
& \cdot\left[-\beta v_{1}^{3}(\sigma)-2 \mu \omega_{1} \Omega \dot{v}_{1}(\sigma)-\left(\sigma_{1}^{2}-2 \Omega \sigma_{1}\right) v_{1}(\sigma)\right. \\
& -\delta \Omega^{2}\left(v_{1}(\sigma) \dot{v}_{1}^{2}(\sigma)+v_{1}^{2}(\sigma) \ddot{v}_{1} \sigma\right)+\xi y_{0} \Omega^{2} \sin \sigma \\
& \left.+\gamma u_{1}\left(\sigma-\tau_{1}\right)\right] \mathrm{d} \sigma+r_{1} \cos t+s_{1} \sin t \\
= & \left(r_{1}-\frac{1}{2} \delta r_{1}^{3}-\frac{1}{2} \delta r_{1} s_{1}^{2}+\frac{3 \beta}{4 \Omega^{2}} r_{1}^{3}+\frac{3 \beta}{4 \Omega^{2}} r_{1} s_{1}^{2}\right. \\
& \left.+\frac{\sigma_{1}^{2}}{\Omega^{2}} r_{1}-\frac{2 \sigma_{1}}{\Omega} r_{1}+\frac{2 \mu \omega_{1} s_{1}}{\Omega}\right) \cos t+\left(-\frac{\delta r_{1}^{3}}{16}\right. \\
& \left.+\frac{3}{16} \delta r_{1} s_{1}^{2}+\frac{\beta r_{1}^{3}}{32 \Omega^{2}}-\frac{3 \beta r_{1} s_{1}^{2}}{32 \Omega^{2}}\right) \cos 3 t+\left(s_{1}\right. \\
& -\frac{1}{2} \delta s_{1}^{3}-\frac{1}{2} \delta s_{1} r_{1}^{2}+\frac{3 \beta}{4 \Omega^{2}} s_{1}^{3}+\frac{3 \beta}{4 \Omega^{2}} s_{1} r_{1}^{2}+\frac{s_{1} \sigma_{1}^{2}}{\Omega^{2}} \\
& \left.-\frac{2 \sigma_{1} s_{1}}{\Omega}-\frac{2 \mu \omega_{1} r_{1}}{\Omega}-y_{0} \xi\right) \sin t+\left(-\frac{3}{16} \delta r_{1}^{2} s_{1}\right. \\
& \left.+\frac{\delta s_{1}^{3}}{16}+\frac{3 \beta r_{1}^{2} s_{1}}{32 \Omega^{2}}-\frac{\beta s_{1}^{3}}{32 \Omega^{2}}\right) \sin 3 t-\frac{s_{2} \gamma \sin \left(t-\tau_{1}\right)}{\Omega^{2}} \\
& -\frac{r_{2} \gamma \cos \left(t-\tau_{1}\right)}{\Omega^{2}},
\end{aligned}
$$

$$
\begin{aligned}
u_{2}(t)= & \frac{1}{\pi \Omega^{2}} \int_{0}^{2 \pi}\left(\frac{1}{2}-\cos (t-\sigma)+\sum_{j=2}^{\infty} \frac{\cos j(t-\sigma)}{1-j^{2}}\right) \\
& \cdot\left[-2 \zeta \omega_{2} \Omega \dot{u}_{1}(\sigma)+2 \Omega\left(\sigma_{1}-\sigma_{2}\right) u_{1}(\sigma)\right. \\
& \left.-\left(\sigma_{1}-\sigma_{2}\right)^{2} u_{1}(\sigma)+\alpha v_{1}\left(\sigma-\tau_{2}\right)\right] \mathrm{d} \sigma \\
& +r_{2} \cos t+s_{2} \sin t \\
= & \left(r_{2}+\frac{\sigma_{1}^{2} r_{2}}{\Omega^{2}}-\frac{2 r_{2} \sigma_{1} \sigma_{2}}{\Omega^{2}}+\frac{r_{2} \sigma_{2}^{2}}{\Omega^{2}}-\frac{2 r_{2} \sigma_{1}}{\Omega}\right. \\
& \left.+\frac{2 r_{2} \sigma_{2}}{\Omega}+\frac{2 s_{2} \zeta \omega_{2}}{\Omega}\right) \cos t-\frac{r_{1} \alpha \cos \left(t-\tau_{2}\right)}{\Omega^{2}} \\
& +\left(s_{2}+\frac{s_{2} \sigma_{1}^{2}}{\Omega^{2}}-\frac{2 s_{2} \sigma_{1} \sigma_{2}}{\Omega^{2}}+\frac{s_{2} \sigma_{2}^{2}}{\Omega^{2}}-\frac{2 s_{2} \sigma_{1}}{\Omega}\right. \\
& \left.+\frac{2 s_{2} \sigma_{2}}{\Omega}-\frac{2 r_{2} \zeta \omega_{2}}{\Omega}\right) \sin t-\frac{s_{1} \alpha \sin \left(t-\tau_{2}\right)}{\Omega^{2}} .
\end{aligned}
$$


Substituting equations (11) and (12) into the solvability conditions equation (A.5) (in Appendix) yields

$$
\begin{gathered}
-\frac{\delta r_{1}^{3}}{2}-\frac{1}{2} \delta r_{1} s_{1}^{2}+\frac{3 \beta r_{1}^{3}}{4 \Omega^{2}}+\frac{3 \beta r_{1} s_{1}^{2}}{4 \Omega^{2}}+\frac{\sigma_{1}^{2} r_{1}}{\Omega^{2}}-\frac{2 \sigma_{1} r_{1}}{\Omega} \\
+\frac{2 \mu \omega_{1} s_{1}}{\Omega}-\frac{r_{2} \gamma \cos \left(\Omega \tau_{1}\right)}{\Omega^{2}}+\frac{s_{2} \gamma \sin \left(\Omega \tau_{1}\right)}{\Omega^{2}}=0, \\
\frac{1}{2} \delta r_{1}^{2} s_{1}+\frac{s_{1}^{3} \delta}{2}+y_{0} \xi-\frac{3 \beta r_{1}^{2} s_{1}}{4 \Omega^{2}}-\frac{3 \beta s_{1}^{3}}{4 \Omega^{2}}-\frac{s_{1} \sigma_{1}^{2}}{\Omega^{2}}+\frac{2 s_{1} \sigma_{1}}{\Omega} \\
+\frac{2 r_{1} \mu \omega_{1}}{\Omega}+\frac{s_{2} \gamma \cos \left(\Omega \tau_{1}\right)}{\Omega^{2}}+\frac{r_{2} \gamma \sin \left(\Omega \tau_{1}\right)}{\Omega^{2}}=0, \\
-\frac{r_{2} \sigma_{1}^{2}}{\Omega^{2}}+\frac{2 r_{2} \sigma_{1} \sigma_{2}}{\Omega^{2}}-\frac{r_{2} \sigma_{2}^{2}}{\Omega^{2}}+\frac{2 r_{2} \sigma_{1}}{\Omega}-\frac{2 r_{2} \sigma_{2}}{\Omega}-\frac{2 s_{2} \zeta \omega_{2}}{\Omega} \\
+\frac{r_{1} \alpha \cos \left(\Omega \tau_{2}\right)}{\Omega^{2}}-\frac{s_{1} \alpha \sin \left(\Omega \tau_{2}\right)}{\Omega^{2}}=0, \\
-\frac{s_{2} \sigma_{1}^{2}}{\Omega^{2}}+\frac{2 s_{2} \sigma_{1} \sigma_{2}}{\Omega^{2}}-\frac{s_{2} \sigma_{2}^{2}}{\Omega^{2}}+\frac{2 s_{2} \sigma_{1}}{\Omega}-\frac{2 s_{2} \sigma_{2}}{\Omega}+\frac{2 r_{2} \zeta \omega_{2}}{\Omega} \\
+\frac{s_{1} \alpha \cos \left(\Omega \tau_{2}\right)}{\Omega^{2}}+\frac{r_{1} \alpha \sin \left(\Omega \tau_{2}\right)}{\Omega^{2}}=0 .
\end{gathered}
$$

For convenience to investigate the dynamics of the whole controlled system, we denote the amplitude of the main system $a_{1}=\sqrt{r_{1}^{2}+s_{1}^{2}}$ and the amplitude of the controller $a_{2}=\sqrt{r_{2}^{2}+s_{2}^{2}}$. To simplify the above four amplitude equations (13)-(16), we set $r_{1}=a_{1} \cos \theta_{1}, s_{1}=a_{1} \sin \theta_{1}, r_{2}=a_{2} \cos \theta_{2}$, $s_{2}=a_{2} \sin \theta_{2}$, and the four amplitude equations yield

$$
\begin{gathered}
\left(-3 a_{1}^{3} \beta-4 a_{1} \sigma_{1}^{2}+8 a_{1} \sigma_{1} \Omega+2 a_{1}^{3} \delta \Omega^{2}\right) \cos \theta_{1} \\
-8 a_{1} \mu \Omega \omega_{1} \sin \theta_{1}+4 a_{2} \gamma \cos \left(\theta_{2}+\Omega \tau_{1}\right)=0, \\
4 y_{0} \xi \Omega^{2}+8 a_{1} \mu \Omega \omega_{1} \cos \theta_{1}+4 a_{2} \gamma \sin \left(\theta_{2}+\Omega \tau_{1}\right) \\
+\left(-3 a_{1}^{3} \beta-4 a_{1} \sigma_{1}^{2}+8 a_{1} \sigma_{1} \Omega+2 a_{1}^{3} \delta \Omega^{2}\right) \sin \theta_{1}=0, \\
\left(-a_{2} \sigma_{1}^{2}+2 a_{2} \sigma_{1} \sigma_{2}-a_{2} \sigma_{2}^{2}+2 a_{2} \sigma_{1} \Omega-2 a_{2} \sigma_{2} \Omega\right) \cos \theta_{2} \\
+a_{1} \alpha \cos \left(\theta_{1}+\Omega \tau_{2}\right)-2 a_{2} \zeta \Omega \omega_{2} \sin \theta_{2}=0,
\end{gathered}
$$

$$
\begin{aligned}
& 2 a_{2} \zeta \Omega \omega_{2} \cos \theta_{2}+a_{1} \alpha \sin \left(\theta_{1}+\Omega \tau_{2}\right)+\left(-a_{2} \sigma_{1}^{2}\right. \\
& \left.\quad+2 a_{2} \sigma_{1} \sigma_{2}-a_{2} \sigma_{2}^{2}+2 a_{2} \sigma_{2} \Omega-2 a_{2} \sigma_{1} \Omega\right) \sin \theta_{2}=0 .
\end{aligned}
$$

To simplify equations (17)-(20), some simple calculations are made in the following:

Equation $(17) \times \cos \theta_{1}+$ equation $(18) \times \sin \theta_{1}$ is

$$
\begin{aligned}
& 4 a_{2} \gamma \cos \left(\theta_{1}-\theta_{2}-\Omega \tau_{1}\right)+4 y_{0} \xi \Omega^{2} \sin \theta_{1}-3 \beta a_{1}^{3} \\
& -4 \sigma_{1}^{2} a_{1}+8 \sigma_{1} \Omega a_{1}+2 \delta \Omega^{2} a_{1}^{3}=0 .
\end{aligned}
$$

Equation $(17) \times \sin \theta_{1}$ - equation $(18) \times \cos \theta_{1}$ is

$$
-2 a_{1} \mu \Omega \omega_{1}-y_{0} \xi \Omega^{2} \cos \theta_{1}+a_{2} \gamma \sin \left(\theta_{1}-\theta_{2}-\Omega \tau_{1}\right)=0
$$

Equation $(19) \times \cos \theta_{2}+$ equation $(20) \times \sin \theta_{2}$ is

$$
-a_{2}\left(\sigma_{1}-\sigma_{2}\right)\left(\sigma_{1}-\sigma_{2}-2 \Omega\right)+a_{1} \alpha \cos \left(\theta_{1}-\theta_{2}+\Omega \tau_{2}\right)=0
$$

Equation $(19) \times \sin \theta_{2}$ - equation $(20) \times \cos \theta_{2}$ is

$$
2 a_{2} \zeta \omega_{2} \Omega+a_{1} \alpha \sin \left(\theta_{1}-\theta_{2}+\Omega \tau_{2}\right)=0 .
$$

Solving equations (21)-(24) and eliminating $\cos \theta_{1}$, $\sin \theta_{1}, \cos \left(\theta_{1}-\theta_{2}\right), \sin \left(\theta_{1}-\theta_{2}\right)$ by means of the relations $\cos ^{2} \theta_{1}+\sin ^{2} \theta_{1}=1, \cos ^{2}\left(\theta_{1}-\theta_{2}\right)+\sin ^{2}\left(\theta_{1}-\theta_{2}\right)=1$ yield the amplitude equations for two possible scenarios.

The uncontrolled system: in this case, the PPF controller does not activate, i.e., $a_{2}=0$; we have the amplitude equation of the excited beam from (21) and (22) in the following:

$$
a_{1}^{2}\left[\left(4 \sigma_{1}\left(\sigma_{1}-2 \Omega\right)+a_{1}^{2}\left(3 \beta-2 \delta \Omega^{2}\right)\right)^{2}+64 \mu^{2} \Omega^{2} \omega_{1}^{2}\right]=16 y_{0} \xi^{2} \Omega^{4} .
$$

The controlled system: in this case, the PPF controller activates, i.e., $a_{2} \neq 0$; then, the amplitude equations of the controlled system are obtained from (21)-(24)

$$
a_{1}^{2} \alpha^{2}=a_{2}^{2}\left[\left(\sigma_{1}-\sigma_{2}\right)^{2}\left(\sigma_{2}-\sigma_{1}+2 \Omega\right)^{2}+4 \zeta^{2} \Omega^{2} \omega_{2}^{2}\right],
$$

$$
\begin{gathered}
16 y_{0}^{3} \xi^{2} \Omega^{4} a_{1}^{2} \alpha^{2}=\left[a_{1}^{2} \alpha\left(4 \sigma_{1}\left(\sigma_{1}-2 \Omega\right)+a_{1}^{2}\left(3 \beta 2 \delta \Omega^{2}\right)\right)\right. \\
-4 a_{2}^{2} \gamma\left(\left(\sigma_{1}-\sigma_{2}\right)\left(\sigma_{1}-\sigma_{2} * 2 \Omega\right) \cos \left(\Omega\left(\tau_{1}+\tau_{2}\right)\right)\right) \\
\left.\left.-2 \zeta \Omega \omega_{2} \sin \left(\Omega\left(\tau_{1}+\tau_{2}\right)\right)\right)\right]^{2}+16\left[2 a_{1}^{2} \alpha \mu \Omega \omega_{1}\right. \\
+a_{2}^{2} \gamma\left(2 \zeta \Omega \omega_{2} \cos \left(\Omega\left(\tau_{1}+\tau_{2}\right)\right)\right. \\
\left.\left.+\left(\sigma_{1}-\sigma_{2}\right)\left(\sigma_{1}-\sigma_{2}-2 \Omega\right) \sin \left(\Omega\left(\tau_{1}+\tau_{2}\right)\right)\right)\right]^{2} .
\end{gathered}
$$

Solving equation (26), we obtain that 


$$
a_{2}^{2}=\frac{a_{1}^{2} \alpha^{2}}{\left(\sigma_{1}-\sigma_{2}\right)^{2}\left(2 \Omega-\sigma_{1}+\sigma_{2}\right)^{2}+4 \zeta^{2} \Omega^{2} \omega_{2}^{2}} .
$$

Substituting (28) into (27), we have the amplitude equation only about $a_{1}$ in the following:

$$
m_{3} a_{1}^{6}+m_{2} a_{1}^{4}+m_{1} a_{1}^{2}+m_{0}=0,
$$

where

$$
\begin{aligned}
m_{0}= & -16 y_{0}^{2} \xi^{2} \Omega^{4}\left[\left(\sigma_{1}-\sigma_{2}\right)^{2}\left(\sigma_{2}-\sigma_{1}+2 \Omega\right)^{2}+4 \zeta^{2} \omega_{2}^{2} \Omega^{2}\right], \\
m_{1}= & 16\left[\alpha^{2} \gamma^{2}+\sigma_{1}^{2}\left(\sigma_{1}-\sigma_{2}\right)^{2}\left(\sigma_{1}-2 \Omega\right)^{2}\left(\sigma_{2}-\sigma_{1}+2 \Omega\right)^{2}\right. \\
& +4 \mu^{2} \Omega^{2} \omega_{1}^{2}\left(\sigma_{1}-\sigma_{2}\right)^{2}\left(\sigma_{2}-\sigma_{1}+2 \Omega\right)^{2} \\
& \left.+4 \zeta^{2} \Omega^{2} \omega_{2}^{2}\left(\sigma_{1}^{2}\left(\sigma_{1}-2 \Omega\right)\right) 2+4 \mu^{2} \Omega^{2} \omega_{1}^{2}\right) \\
& -2 \alpha \gamma\left(\sigma_{1}\left(\sigma_{2}-\sigma_{2}\right)\left(\sigma_{1}-2 \Omega\right)\left(\sigma_{1}-\sigma_{2}-2 \Omega\right)\right. \\
& +4 \alpha \gamma \Omega\left(\mu \omega_{1}\left(\sigma_{1}-\sigma_{2}\right)\left(\sigma_{1}-\sigma_{2}-2 \Omega\right)\right. \\
& \left.-4 \zeta \mu \Omega^{2} \omega_{1} \omega_{2}\right) \cos \left(\Omega\left(\tau_{1}+\tau_{2}\right)\right) \\
& \left.\left.+\zeta \sigma_{1} \omega_{2}\left(\sigma_{1}-2 \Omega\right)\right) \sin \left(\Omega\left(\tau_{1}+\tau_{2}\right)\right)\right], \\
m_{2}= & 8\left(3 \beta-2 \delta \Omega^{2}\right)\left[\sigma _ { 1 } ( \sigma _ { 1 } - 2 \Omega ) \left(( \sigma _ { 1 } - \sigma _ { 2 } ) ^ { 2 } \left(\sigma_{2}-\sigma_{1}\right.\right.\right. \\
& \left.+2 \Omega)^{2}+4 \zeta^{2} \Omega^{2} \omega_{2}^{2}\right)-\alpha \gamma\left(\sigma_{1}-\sigma_{2}\right)\left(\sigma_{1}-\sigma_{2}\right. \\
& \left.-2 \Omega) \cos \left(\Omega\left(\tau_{1}+\tau_{2}\right)\right)+2 \alpha \gamma \zeta \Omega \omega_{2} \sin \left(\Omega\left(\tau_{1}+\tau_{2}\right)\right)\right], \\
m_{3}= & \left(3 \beta-2 \delta \Omega^{2}\right)^{2}\left[\left(\sigma_{1}-\sigma_{2}\right)^{2}\left(\sigma_{2}-\sigma_{1}+2 \Omega\right)^{2}\right. \\
& \left.+4 \zeta^{2} \Omega^{2} \omega_{2}^{2}\right] .
\end{aligned}
$$

\section{Stability of Periodic Solutions}

4.1. Stability of Periodic Solutions of the Uncontrolled System. For the uncontrolled beam, i.e., $r_{2}=0, s_{2}=0, \gamma=0, \alpha=0$, we obtain the second approximation from equation (11) as

$$
v_{2}(t)=r_{1} \cos t+s_{1} \sin t
$$

In the above equation (31), we have omitted the higher order terms $\cos 3 t, \sin 3 t$ because of the weak nonlinearities. To study the stability of the periodic solution of the uncontrolled beam, we first perturb the periodic solution $v_{2}(t)$ in equation (31) by introducing disturbance terms $z(t)$. Replacing $v_{2}(t)$ by $v_{2}(t)+z(t)$ in equation (1) and linearizing on $z(t)$, we get the linear variational equation in the following:

$$
\begin{aligned}
\ddot{z}+z= & \frac{1}{\Omega^{2}}\left[-2 \mu \omega_{1} \Omega \dot{z}-\left(\sigma_{1}^{2}-2 \Omega \sigma_{1}\right) z-3 \beta v_{2}^{2} z\right. \\
& \left.-\delta \Omega^{2}\left(2 v_{2} \dot{v}_{2} \dot{z}+\dot{v}_{2}^{2} z\right)-\delta \Omega^{2}\left(v_{2}^{2} \ddot{z}+2 v_{2} \ddot{v}_{2} z\right)\right] .
\end{aligned}
$$

Corresponding to the Floquet theory, the solution of equation (32) is written as

$$
z(t)=\exp (\rho t) Z(t)
$$

and equation (32) is transformed into

$$
\begin{aligned}
\ddot{Z}+Z=[ & \frac{1}{2}\left(r_{1}^{2}+s_{1}^{2}\right) \delta-\frac{1}{2}\left(2+r_{1}^{2} \delta+s_{1}^{2} \delta\right) \rho^{2} \\
& -\frac{3\left(r_{1}^{2}+s_{1}^{2}\right) \beta}{2 \Omega^{2}}-\frac{\sigma_{1}^{2}-2 \sigma_{1} \Omega+2 \mu \rho \Omega \omega_{1}}{\Omega^{2}} \\
& +\frac{1}{2 \Omega^{2}}\left(-4 r_{1} s_{1} \delta \rho \Omega^{2}+\left(s_{1}^{2}-r_{1}^{2}\right)\left(3 \beta-3 \delta \Omega^{2}\right.\right. \\
& \left.\left.+\delta \rho^{2} \Omega^{2}\right)\right) \cos 2 t+\frac{1}{\Omega^{2}}\left(\left(r_{1}^{2}-s_{1}^{2}\right) \delta \rho \Omega^{2}\right. \\
& \left.\left.-r_{1} s_{1}\left(3 \beta-3 \delta \Omega^{2}+\delta \rho^{2} \Omega^{2}\right)\right) \sin 2 t\right] Z_{1} \\
& +\left[-\frac{1}{\Omega}\left(\left(2+r_{1}^{2} \delta+s_{1}^{2} \delta\right) \rho \Omega+2 \mu \omega_{1}\right)+\delta\left(-2 r_{1} s_{1}\right.\right. \\
& \left.-r_{1}^{2} \rho+s_{1}^{2} \rho\right) \cos (2 t)+\delta\left(r_{1}^{2}-s_{1}^{2}\right. \\
& \left.\left.-2 r_{1} s_{1} \rho\right) \sin 2 t\right] \dot{Z}-\left[\frac{1}{2}\left(r_{1}^{2}+s_{1}^{2}\right) \delta+\frac{1}{2}\left(r_{1}^{2}\right.\right. \\
& \left.\left.+s_{1}^{2}\right) \delta \cos (2 t)+r_{1} s_{1} \delta \sin (2 t)\right] \ddot{Z} .
\end{aligned}
$$

The first approximation of equation (34) can be expressed as

$$
Z(t)=a_{0}+a_{1} \cos t+a_{2} \sin t .
$$

Substituting equation (35) into equation (34) and equating the constant terms and the coefficients of the same harmonic terms, we obtain the following set of linear homogeneous algebraic equations governing the coefficients $a_{0}, a_{1}, a_{2}$ :

$$
\begin{aligned}
k_{1} a_{0} & =0, \\
k_{2} a_{1}+k_{3} a_{2} & =0, \\
k_{4} a_{1}+k_{5} a_{2} & =0,
\end{aligned}
$$


where

$$
\begin{aligned}
& k_{1}=1+\rho^{2}+\frac{\sigma_{1}^{2}}{\Omega^{2}}-\frac{2 \sigma_{1}}{\Omega}+\frac{2 \mu \rho \omega_{1}}{\Omega} \\
& +\frac{1}{2 \Omega^{2}}\left(r_{1}^{2}+s_{1}^{2}\right)\left(3 \beta-\delta \Omega^{2}+\delta \rho^{2} \Omega^{2}\right) \\
& k_{2}=\frac{1}{4 \Omega^{2}}\left(3 r_{1}^{2}+s_{1}^{2}\right)\left(3 \beta-2 \delta \Omega^{2}+\delta \rho^{2} \Omega^{2}\right)+\rho^{2} \\
& +\frac{\sigma_{1}^{2}}{\Omega^{2}}-\frac{2 \sigma_{1}}{\Omega}+\frac{2 \mu \rho \omega_{1}}{\Omega} \\
& k_{3}=2 \rho+\frac{2 \mu \omega_{1}}{\Omega}+\left(r_{1}^{2}+s_{1}^{2}\right) \delta \rho \\
& +\frac{1}{2 \Omega^{2}} r_{1} s_{1}\left(3 \beta-2 \delta \Omega^{2}+\delta \rho^{2} \Omega^{2}\right) \\
& k_{4}=-2 \rho-\frac{2 \mu \omega_{1}}{\Omega}-\left(r_{1}^{2}+s_{1}^{2}\right) \delta \rho \\
& +\frac{1}{2 \Omega^{2}} r_{1} s_{1}\left(3 \beta-2 \delta \Omega^{2}+\delta \rho^{2} \Omega^{2}\right) \\
& k_{5}=\frac{1}{4 \Omega^{2}}\left(3 s_{1}^{2}+r_{1}^{2}\right)\left(3 \beta-2 \delta \Omega^{2}+\delta \rho^{2} \Omega^{2}\right)+\rho^{2}+\frac{\sigma_{1}^{2}}{\Omega^{2}} \\
& -\frac{2 \sigma_{1}}{\Omega}+\frac{2 \mu \rho \omega_{1}}{\Omega}
\end{aligned}
$$

Since $a_{0}, a_{1}, a_{2}$ are not all zero, the following coefficients matrix must be zero; i.e.,

$$
\left|\begin{array}{lll}
k_{1} & 0 & 0 \\
0 & k_{2} & k_{3} \\
0 & k_{4} & k_{5}
\end{array}\right|=0 .
$$

Expanding this determinant yields

$$
f_{0}^{*} \rho^{6}+f_{1}^{*} \rho^{5}+f_{2}^{*} \rho^{4}+f_{3}^{*} \rho^{3}+f_{4}^{*} \rho^{2}+f_{5}^{*} \rho+f_{6}^{*}=0 .
$$

This is a nonlinear algebraic equation. In this case, the Routh-Hurwitz criterion can be utilized to study the stability of the periodic solution.

4.2. Stability of Periodic Solutions of the Controlled System. To investigate the stability of the periodic solution of the PPF controlled system (2), we need to examine the behavior of the small perturbations from the steady state solutions $v_{2}(t), u_{2}(t)$ in equations (11) and (12). Thus, we assume that

$$
\begin{aligned}
& v(t)=v_{2}(t)+z_{1}(t), \\
& u(t)=u_{2}(t)+z_{2}(t),
\end{aligned}
$$

where $z_{1}(t)$ and $z_{2}(t)$ are small perturbations. Substituting equation (40) into equation (2) and linearizing on $z_{1}(t)$ and $z_{2}(t)$ yield

$$
\begin{aligned}
\ddot{z}_{1}+z_{1}= & \frac{1}{\Omega^{2}}\left[-2 \mu \omega_{1} \Omega \dot{z}_{1}-\left(\sigma_{1}^{2}-2 \Omega \sigma_{1}\right) z_{1}-3 \beta v_{2}^{2} z_{1}\right. \\
& -\delta \Omega^{2}\left(2 v_{2} \dot{v}_{2} \dot{z}_{1}+\dot{v}_{2}^{2} z_{1}+2 v_{2} \ddot{v}_{2} z_{1}+v_{2}^{2} \ddot{z}_{1}\right) \\
& \left.+\gamma z_{2}\left(t-\tau_{1}\right)\right] \\
\ddot{z}_{2}+z_{2}= & \frac{1}{\Omega^{2}}\left[-2 \zeta \omega_{2} \Omega \dot{z}_{2}+2 \Omega\left(\sigma_{1}-\sigma_{2}\right) z_{2}\right. \\
& \left.-\left(\sigma_{1}-\sigma_{2}\right)^{2} z_{2}+\alpha z_{1}\left(t-\tau_{2}\right)\right] .
\end{aligned}
$$

According to Floquet theory, equation (41) admits solutions of the form

$$
z_{i}(t)=\exp (\rho t) Z_{i}(t), \quad i=1,2,
$$

where $Z_{i}(t)$ is a periodic function with period $T=2 \pi$, which is equal to the period of $v(t), u(t)$. Substituting equation (42) into equation (41) yields

$$
\begin{aligned}
\ddot{Z}_{1}+Z_{1}= & \frac{1}{2}\left(r_{1}^{2}+s_{1}^{2}\right) \delta-\frac{3 \beta}{2 \Omega^{2}}\left(r_{1}^{2}+s_{1}^{2}\right)-\frac{\sigma_{1}^{2}}{\Omega^{2}}+\frac{2 \sigma_{1}}{\Omega} \\
& -\frac{2 \mu \rho \omega_{1}}{\Omega}-\frac{1}{2}\left(2+\delta r_{1}^{2}+\delta s_{1}^{2}\right) \rho^{2} \\
& +\frac{1}{2 \Omega^{2}}\left(-4 r_{1} s_{1} \delta \rho \Omega^{2}+\left(s_{1}^{2}-r_{1}^{2}\right)(3 \beta\right. \\
& \left.\left.+\delta\left(\rho^{2}-3\right) \Omega^{2}\right)\right) \cos (2 t)+\frac{1}{\Omega^{2}}\left(\left(r_{1}^{2}-s_{1}^{2}\right) \delta \rho \Omega^{2}\right. \\
& \left.\left.-r_{1} s_{1}\left(3 \beta+\delta \Omega^{2}\left(\rho^{2}-3\right)\right)\right) \sin (2 t)\right] Z_{1}(t) \\
& +\frac{\gamma}{\Omega^{2}} \exp \left(-\rho \tau_{1}\right) Z_{2}\left(t-\tau_{1}\right)+\left[-\left(2+r_{1}^{2} \delta+s_{1}^{2} \delta\right) \rho\right. \\
& -\frac{2 \mu \omega_{1}}{\Omega}+\delta\left(-2 r_{1} s_{1}-r_{1}^{2} \rho+s_{1}^{2} \rho\right) \cos (2 t) \\
& \left.+\delta\left(r_{1}^{2}-s_{1}^{2}-2 r_{1} s_{1} \rho\right) \sin (2 t)\right] \dot{Z}_{1}+\left[-\frac{1}{2} \delta\left(r_{1}^{2}\right.\right. \\
& \left.\left.+s_{1}^{2}\right)-\frac{1}{2} \delta\left(r_{1}^{2}-s_{1}^{2}\right) \cos (2 t)-r_{1} s_{1} \delta \sin (2 t)\right] \ddot{Z}_{1},
\end{aligned}
$$

$$
\begin{aligned}
\ddot{Z}_{2}+Z_{2}= & \frac{\alpha \exp \left(-\rho \tau_{2}\right)}{\Omega^{2}} Z_{1}\left(t-\tau_{2}\right)+\left(-\rho^{2}-\frac{\sigma_{1}^{2}}{\Omega^{2}}\right. \\
& \left.+\frac{2 \sigma_{1} \sigma_{2}}{\Omega^{2}}-\frac{\sigma_{2}^{2}}{\Omega^{2}}+\frac{2 \sigma_{1}}{\Omega}-\frac{2 \sigma_{2}}{\Omega}-\frac{2 \zeta \rho \omega_{2}}{\Omega}\right) Z_{2} \\
& -\left(2 \rho+\frac{2 \zeta \omega_{2}}{\Omega}\right) \dot{Z}_{2} .
\end{aligned}
$$

The first-order approximate solutions of equations (43) and (44) can be expressed as

$$
\begin{aligned}
& Z_{1}(t)=p_{0}+p_{1} \cos t+p_{2} \sin t \\
& Z_{2}(t)=p_{3}+p_{4} \cos t+p_{5} \sin t .
\end{aligned}
$$


Substituting (45) and (46) into (43) and (44) and equating the coefficients of the same harmonic terms yield a set of linear homogeneous algebraic equations governing the coefficients $p_{0}, p_{1}, p_{2}, p_{3}, p_{4}$, and $p_{5}$. Setting the determinant of the coefficient matrix equal to zero, we obtain the so-called Hill's determinant governing $\rho$. Expanding this determinant yields

$$
\begin{aligned}
& b_{12} \rho^{12}+b_{11} \rho^{11}++\cdots+b_{2} \rho^{2}+b_{1} \rho+b_{0}+\left[\left(d_{c 0}\right.\right. \\
& \left.+d_{c 1} \rho+d_{c 2} \rho^{2}+\cdots+d_{c 7} \rho^{7}+d_{c 8} \rho^{8}\right) \cos \left(\Omega\left(\tau_{1}+\tau_{2}\right)\right) \\
& +\left(d_{s 0}+d_{s 1} \rho+d_{s 2} \rho^{2}+\cdots+d_{s 6} \rho^{6}\right. \\
& \left.\left.+d_{s 7} \rho^{7}\right) \sin \left(\Omega\left(\tau_{1}+\tau_{2}\right)\right)\right] \exp \left(-\rho \Omega\left(\tau_{1}+\tau_{2}\right)\right) \\
& +\left[\left(e_{c 4} \rho^{4}+e_{c 3} \rho^{3}+e_{c 2} \rho^{2}+e_{c 1} \rho+e_{c 0}\right) \cos \left(\Omega\left(\tau_{1}+\tau_{2}\right)\right)\right. \\
& +\left(e_{s 3} \rho^{3}+e_{s 2} \rho^{2}+e_{s 1} \rho\right. \\
& \left.\left.+e_{s 0}\right) \sin \left(\Omega\left(\tau_{1}+\tau_{2}\right)\right)\right] \exp \left(-2 \rho \Omega\left(\tau_{1}+\tau_{2}\right)\right) \\
& +c_{0} \exp \left(-3 \rho \Omega\left(\tau_{1}+\tau_{2}\right)\right)=0 .
\end{aligned}
$$

Equation (47) can be solved numerically. The approximate solutions $v_{2}(t), u_{2}(t)$ in equations (11) and (12) are asymptotically stable if and only if all of the eigenvalues $\rho$ lie in the left half of the complex plane, and they are unstable if at least one eigenvalue lies in the right half-plane. The solution subsequent to the bifurcation depends on the manner in which the eigenvalues cross from the left half-plane to the right $[22,23]$.

\section{Results and Discussions}

In this section, the steady-state response of the nonlinear beam and the PPF controller is studied analytically and numerically. The parameters are fixed at $\xi=0.89663, \omega_{1}=$ $3.06, \mu=0.05, \beta=14.41, \delta=3.27, y_{0} \in(0,0.1), \quad \zeta=0.001$, $\alpha=3, \gamma=3, \tau_{1}=0, \tau_{2}=0, \sigma_{2}=0, \sigma_{1}=0$, unless otherwise specified. These parameters are derived from the previously published works $[3-5,14,16,18,21]$. The main results are presented in graphical forms and tables, such that the solid lines stand for stable solutions, the dashed lines stand for unstable solutions, and the red points stand for numerical solutions.

5.1. Time Delay Stability Margin for Different Values of Various Control Parameters. In this subsection, our efforts are focused on determining the maximum limits of time delays at which the system solution remains stable. In [16], the authors named these limits of time delays as "time delay stability margin." According to equations (27) and (47), we infer that both the amplitudes and stability of the steady state response of equation (2) are affected by the sum of delays $\tau_{1}+\tau_{2}$. Therefore, the time delay stability margin depends on the overall delay $\tau_{1}+\tau_{2}$. This result is consistent with the previously published work [16]. Figure 1 shows time histories of the main system and the controller for four different cases, $\quad$ i.e., $\quad \tau_{1}=0, \tau_{2}=0.05 ; \quad \tau_{1}=0.01, \tau_{2}=0.04$; $\tau_{1}=0.04, \tau_{2}=0.01 ; \tau_{1}=0.03, \tau_{2}=0.02$. We observe that the amplitudes of the main system and the controller are almost the same in four different cases. Figure 2 presents a comparison of time delay $\tau_{1}$-response curves between numerical simulations and approximate solutions. It can be seen that the approximate solutions are in good agreement with numerical simulations.

Figure 3 shows time delay stability margin under different parameters. Figures 3(a1) and (b1) illustrate that time delay stability margin will hardly change when $y_{0}$ varies in the range $(0,0.1)$. It can be seen that the steady state response of the whole system is stable when $\tau_{1}+\tau_{2}$ locates in the interval $(0,0.1)$. Beyond this range, the whole system becomes unstable. From Figures 3(a2) to (b4), the parameter $y_{0}$ is fixed at 0.07. Figures 3(a2), (b2) and (a3), (b3) illustrate that increasing the feedback signal gain $\alpha$ and the control signal gain $\gamma$ can shrink the stable region. From these figures, we can see that the controlled system is always stable in the time delay interval $(0,0.08)$ when the control signal gain $\gamma$ and the feedback signal gain $\alpha$ vary in the interval $(0,5)$. From Figures 3(a4) and (b4), we observe that increasing the damping coefficient $\zeta$ can broaden the stable region of the solution while decreasing $\zeta$ shrinks the stable region. Based on these figures, we conclude that the unavoidable total time delay $\tau_{1}+\tau_{2}$ in the controlled system should not exceed 0.08 .

Figures 4 and 5 show time histories of the main system and the controller for different values of time delays $\tau_{1}, \tau_{2}$. Figure 4 shows stable behavior for the main system and the controller at $\tau_{1}=0.03, \tau_{2}=0.04$. It can be seen that the whole system passes through a transient region into a stable steady-state region. Figure 5 shows a complex unstable motion for the system and the controller when the total time delay $\tau_{1}+\tau_{2}$ exceeds the stable region (i.e., $\left.\tau_{1}=0.1, \tau_{2}=0.04\right)$.

5.2. Effects of Control Parameters on the Existence of MultipleSolution Region. In this subsection, we investigate the effects of various parameters on the existence of multiple-solution region for equation (2). For convenience's sake, we denote $A_{1}=a_{1}^{2}$ in equation (29) and rewrite equation (29) in the form

$$
m_{3} A_{1}^{3}+m_{2} A_{1}^{2}+m_{1} A_{1}+m_{0}=0 .
$$

Based on Girolamo Cardano, we give the discriminant of roots in the following:

Case 1: if $1 / 4\left(27 m_{3}^{2} m_{0}-9 m_{3} m_{2} \quad m_{1}+2 m_{2}^{3}\right)^{2}+$ $\left(3 m_{3} m_{1}-m_{2}^{2}\right)^{3}<0$ and $\left(m_{2} / m_{3}\right)<0,\left(m_{1} / m_{3}\right)>0$, $\left(m_{0} / m_{3}\right)<0$, then equation (48) has three different positive real roots which means equation (29) has three different amplitudes, and system (2) has tristable state vibration.

Case 2: if $1 / 4\left(27 m_{3}^{2} m_{0}-9 m_{3} m_{2} \quad m_{1}+2 m_{2}^{3}\right)^{2}+$ $\left(3 m_{3} m_{1}-m_{2}^{2}\right)^{3}=0$ and $\left(3 m_{3} m_{1}-m_{2}^{2}\right) \neq 0,\left(27 m_{3}^{2} m_{0}-\right.$ $\left.9 m_{3} m_{2} m_{1}+2 m_{2}^{3}\right) \neq 0,\left(m_{2} / m_{3}\right)<0,\left(m_{1} / m_{3}\right)>0,\left(m_{0} / m_{3}\right)$ $<0$, then equation (48) has three positive real roots, two of which are equal. It means equation (29) has two different amplitudes and system (2) has bistable steady- 


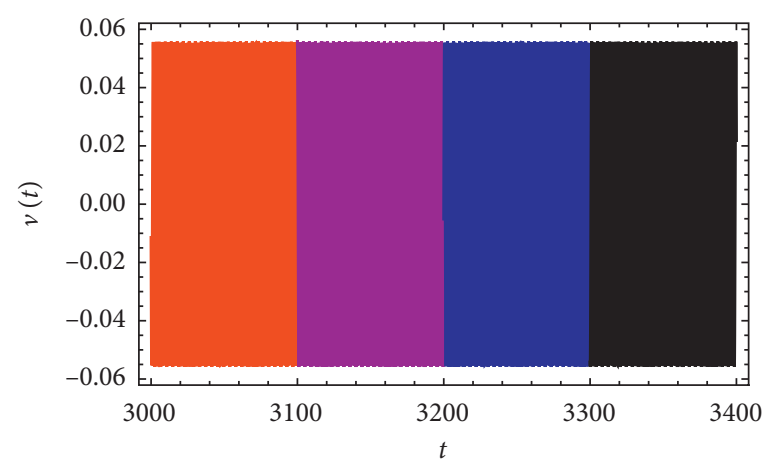

(a)

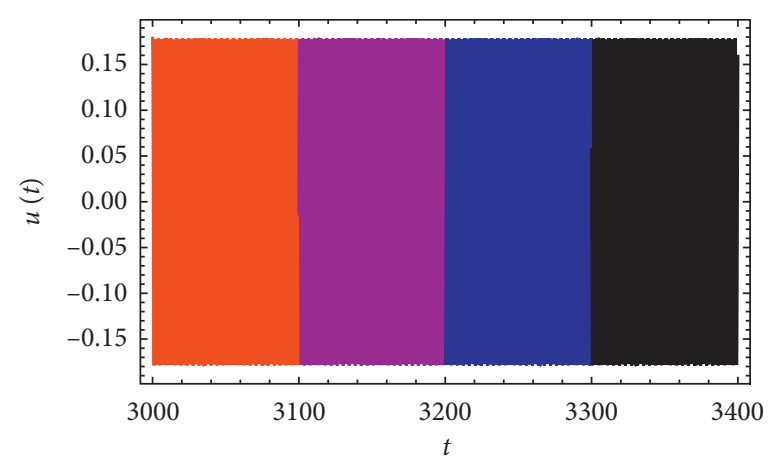

(b)

Figure 1: Time history for the controlled system when $\sigma_{1}=0, \sigma_{2}=0, \zeta=0.001, y_{0}=0.07 ; 3000 \leq t \leq 3100, \tau_{1}=0, \tau_{2}=0.05$; $3100 \leq t \leq 3200, \tau_{1}=0.01, \tau_{2}=0.04 ; 3200 \leq t \leq 3300, \tau_{1}=0.04, \tau_{2}=0.01 ;$ and $3300 \leq t \leq 3400, \tau_{1}=0.03, \tau_{2}=0.02$ : (a) the main system; (b) the controller.

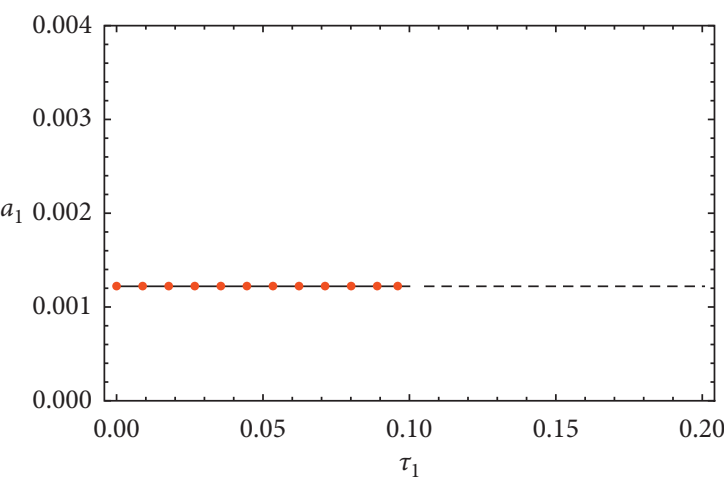

(a)

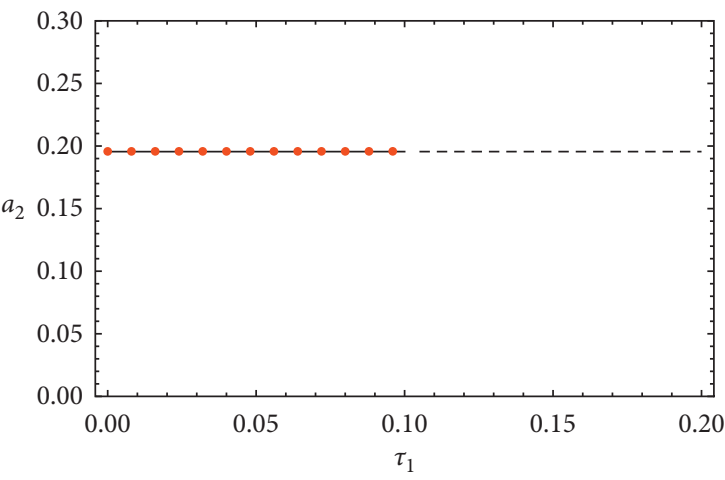

(b)

FIGURE 2: Comparison of time delay $\tau_{1}$-response curves between numerical simulations and approximate solutions when $\tau_{2}=0, y_{0}=0.07, \alpha=3, \gamma=3, \zeta=0.001$, and $\sigma_{1}=\sigma_{2}=0$ : (a) the main system; (b) the controller.

state vibration. This is the critical case of transition from monostable state to tristable state, which often means the occurrence of saddle node bifurcation, and jumping phenomena occur at these points.

Case 3: except for the above two cases, equation (48) has only one positive real root; i.e., equation (29) has one amplitude and system (2) has simple steady-state vibration.

Figure 6 shows the existence of multiple-solution region in $y_{0}-\sigma_{1}$ plane for the uncontrolled system, the controlled system without time delay, and the controlled system with $\tau_{1}+\tau_{2}=0.03$. The blank region represents only one solution. The red dashed line boundary stands for two different solutions and the blue region stands for three different solutions. Figure 6(a) illustrates that there are no multiple solutions for the uncontrolled system when $y_{0}$ varies in the interval $(0,0.1)$. That is to say, although the uncontrolled beam is a nonlinear beam, it does not show the nonlinear characteristics under such parameters. Figure 6(b) shows that the controlled system without time delay begins to have multiple solutions when $y_{0}>0.048$, and the larger $y_{0}$ is, the wider the multiple-solution region is. It means that the controlled system makes the linear system nonlinear even when there is no time delay. Figure 6(c) shows that the multiple-solution region of the controlled system with $\tau_{1}+\tau_{2}=0.03$ is obviously increased compared with the first two cases. Even if the force amplitude $y_{0}$ is very small, the controlled system shows obvious non-linear characteristics.

Figure 7 shows the existence of multiple-solution region in $\tau-\sigma_{1}$ plane for the controlled system with $\zeta=0.05$ and $\zeta=0.001$, respectively. Figure 7(a) illustrates that when the total delay $\tau_{1}+\tau_{2}>0.13$, the controlled system with $\zeta=0.05$ has multiple solutions. That is to say, despite the appearance of time delay, the controlled system with $\zeta=0.05$ cannot exhibit the non-linear characteristics when $\tau_{1}+\tau_{2}<0.13$. Figure 7(b) shows that the multiple-solution region with $\zeta=$ 0.001 increases significantly compared with the controlled system with $\zeta=0.05$.

Figures 8-10 show the effect of control parameters $\gamma, \alpha$, and $\zeta$ on the existence of multiple-solution region. From Figures 8(a) and 9(a), we observe that increasing $\gamma$ and $\alpha$ leads to a slight broadening of the multiple-solution region 

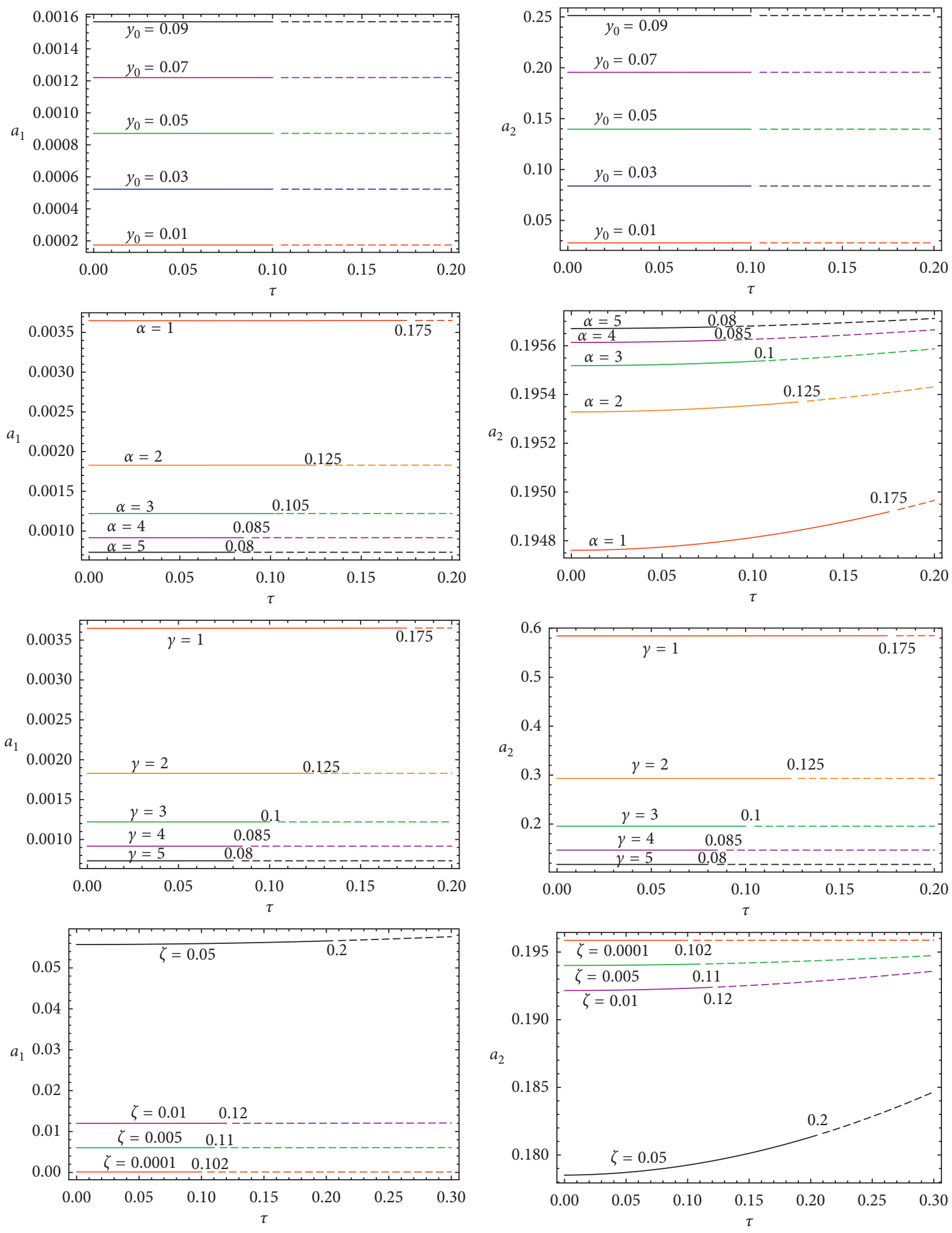

(a)

(b)

FIgURE 3: Time delay $\tau$-response curves of the PPF controlled system where $\tau=\tau_{1}+\tau_{2}$ : (a) the main system; (b) the controller.

for the controlled system without time delay. Figures 8 (b) and 9(b) illustrate that the increase of $\gamma$ and $\alpha$ leads to a significant broadening of the multiple-solution region for the controlled system with $\tau_{1}+\tau_{2}=0.03$. Figure 10 (a) shows that the controlled system without time delay has multiple solutions only when the damping coefficient $\zeta<0.006$. When the total time delay $\tau_{1}+\tau_{2}=0.03$, the multiple-solution region of the controlled system is significantly larger than that of the former in Figure 10(b). From Figure 10(b), we also observe that even if the total time delay 


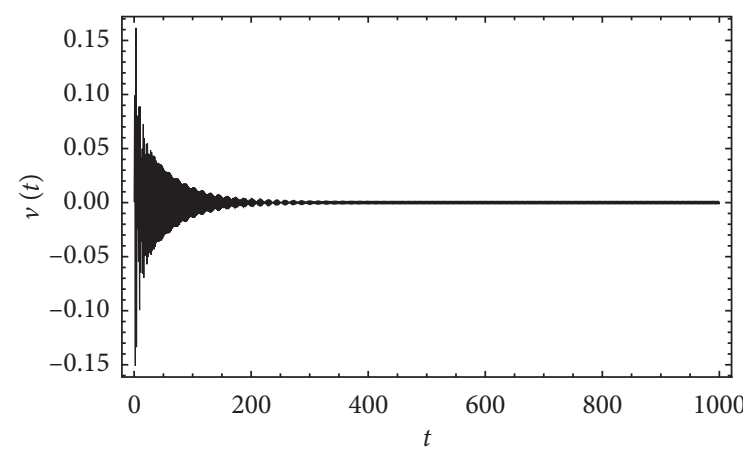

(a)

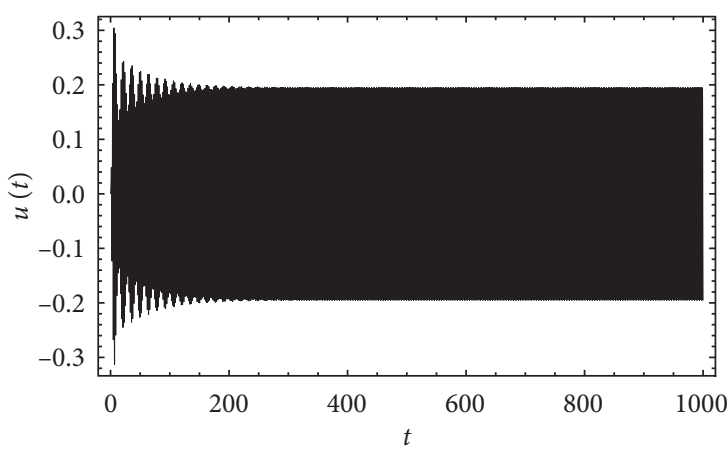

(b)

Figure 4: Time history of the controlled system when $\tau_{1}=0.03, \tau_{2}=0.04, y_{0}=0.07$ with the initial condition $v(0)=0.001, u(0)=0.001, \dot{v}(0)=0.1$, and $\dot{u}(0)=0.01$ : (a) the main system; (b) the controller.

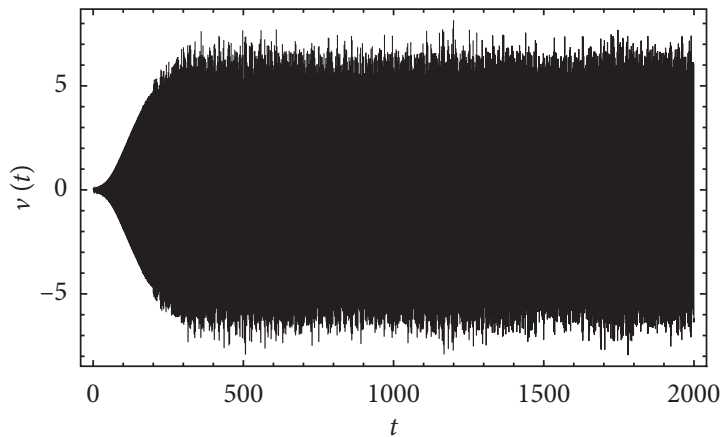

(a)

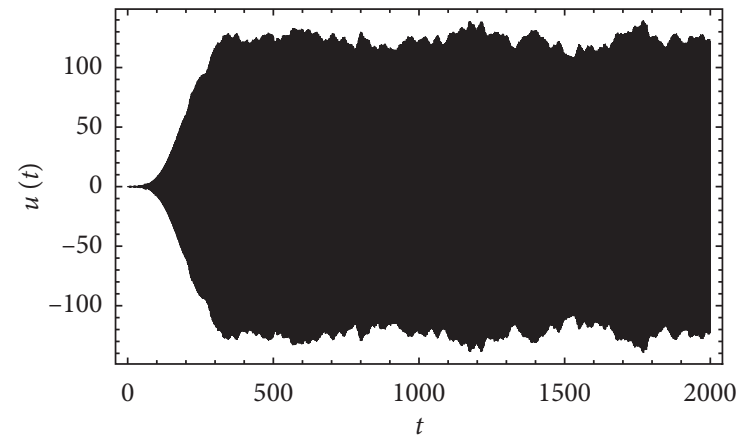

(b)

Figure 5: Time history of the controlled system when $\tau_{1}=0.1, \tau_{2}=0.04, y_{0}=0.07$ with the initial condition $v(0)=0.001, u(0)=0.001, \dot{v}(0)=0.1$, and $\dot{u}(0)=0.01$ : (a) the main system; (b) the controller.

is not zero, the controlled system has multiple solutions only when the damping coefficient is less than 0.016 .

Figure 11 shows the frequency response curves for the uncontrolled system. A comparison between the approximations obtained by the integral iterative method and numerical simulations is made to verify the analytical results in Figure 11(a). Figure 11(b) illustrates that the steady-state amplitude of uncontrolled system is a monotonic increasing function with respect to the force amplitude $y_{0}$. As the force amplitude $y_{0}$ increases in the range $(0,0.1)$, the curve is bent to the left indicating a soft effect. However, the jump phenomenon does not occur. It means that the nonlinearity does not work when $y_{0} \in(0,0.1)$.

Figures 12 and 13 study the frequency $\sigma_{1}$-response curves of the controlled system with $\tau_{1}=0.02, \tau_{2}=0.01$ at $\zeta=0.05$ and $\zeta=0.001$, respectively. In addition, we observe that the numerical results correspond well with the theoretical results for both cases from these figures. Figure 12 shows that the controlled system with $\tau_{2}=0.02, \tau_{1}=0.01, \zeta=0.05$ is always stable and never has jump phenomenon throughout the range $\sigma_{1} \in(-0.5,0.5)$. Figure 13 illustrates that the controlled system with $\tau_{2}=$ $0.02, \tau_{1}=0.01, \zeta=0.001$ has three solutions in the range
$(0.05,0.3)$. In addition, we observe that the larger two solutions are unstable and the smallest solution is stable in the range $(0.05,0.18)$. Within the range $(0.18,0.3)$, the intermediate solution is unstable and the other two solutions are stable. The jump phenomenon occurs at $\sigma_{1}=0.3$. It means that there is bistability in the range $\sigma_{1} \in(0.18,0.3)$. Figures 14 and 15 confirm the coexistence of two stable solutions at $\tau_{1}=0.02, \tau_{1}=0.01, \zeta=0.001$ with two different initial conditions.

In summary, the appearance of time delay and near zero damping coefficient $\zeta$ are two main factors leading to the nonlinear characteristics of the controlled system. Perhaps, we can adjust different initial values to achieve the effect of vibration reduction in the region where multiple solutions exist.

5.3. The Location of the Minimum Steady-State Main System Amplitude. The previously published works $[5,7-10,16]$ pointed out that the minimum steady-state amplitude of the main system occurred at $\sigma_{1}=\sigma_{2}$ for the PPF controlled system. In this section, our efforts are focused on finding out where the minimum amplitude $a_{1}$ occurs. From equations (26)-(29), we conclude that the vibration of the main system 


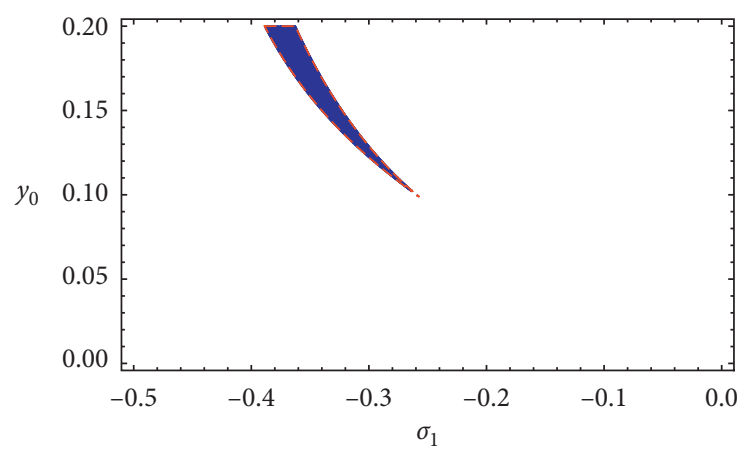

Blank region: single solution area Red dashed line: double solution area Blue region: triple solution area

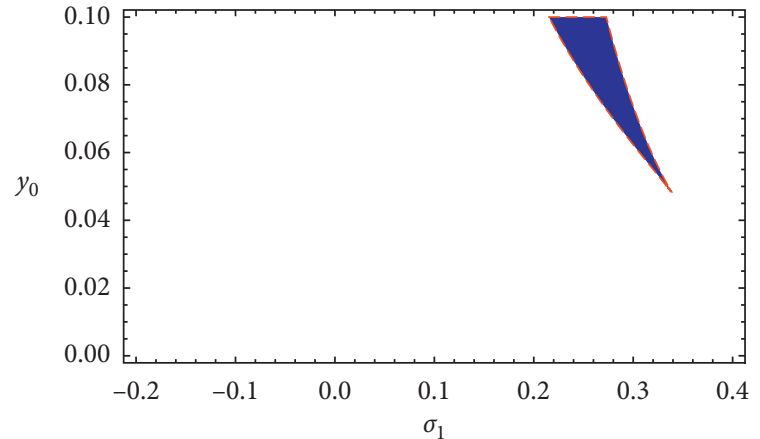

Blank region: single solution area Red dashed line: double solution area Blue region: triple solution area

(a)

(b)

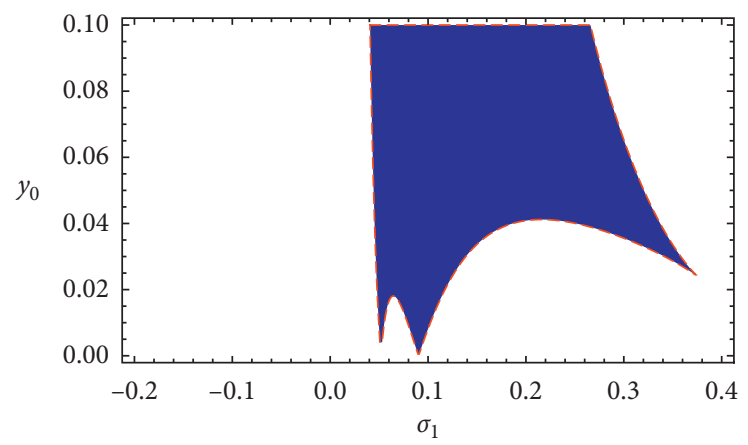

Blank region: single solution area

Red dashed line: double solution area

Blue region: triple solution area

(c)

FIgURE 6: The system solution behavior in $y_{0}-\sigma_{1}$ plane: (a) the uncontrolled system; (b) the controlled system without time delay; (c) the controlled system with $\tau=\tau_{1}+\tau_{2}=0.03$.

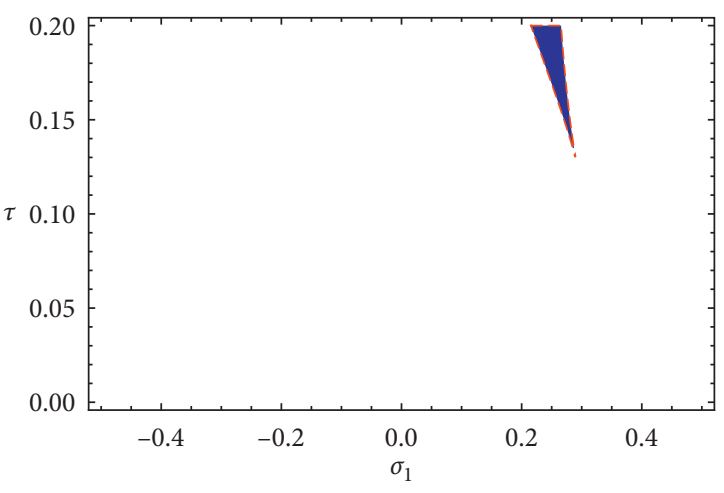

(a)

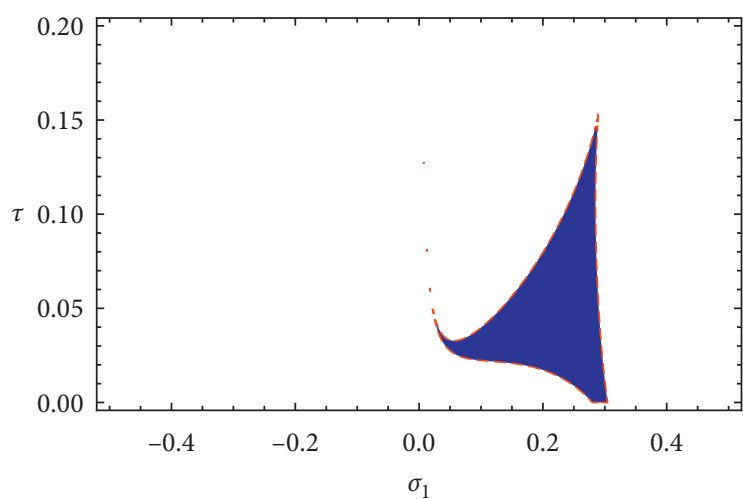

(b)

FIgURe 7: The system solution behavior in $\tau-\sigma_{1}$ plane when $y_{0}=0.07$ : (a) $\zeta=0.05$; (b) $\zeta=0.001$.

vanishes when $\sigma_{1}=\sigma_{2}$ and $\zeta=0$. Equation (31) is only an amplitude equation on $a_{1}$. According to the Theorem of the Existence of Implicit Functions, the amplitude $a_{1}$ in equation (31) can be considered as a function on $\Omega$. Owing to the relation $A_{1}=a_{1}^{2}$ and $a_{1} \geq 0$, the minimum point of $a_{1}$ is equivalent to that of $A_{1}$. According to calculus theory, the minimum point of amplitude $A_{1}$ must satisfy the following equation set: 


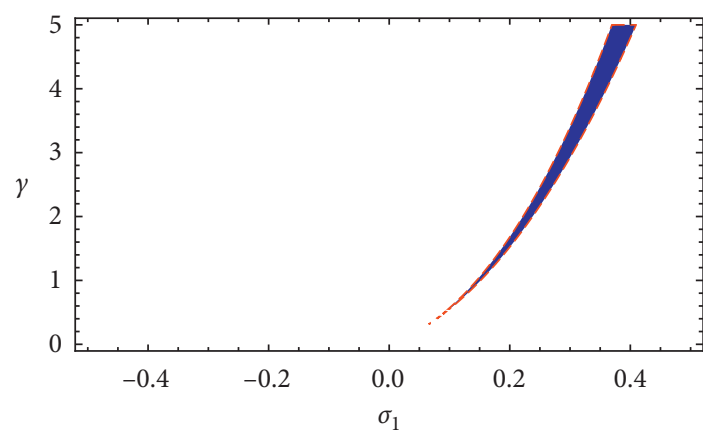

(a)

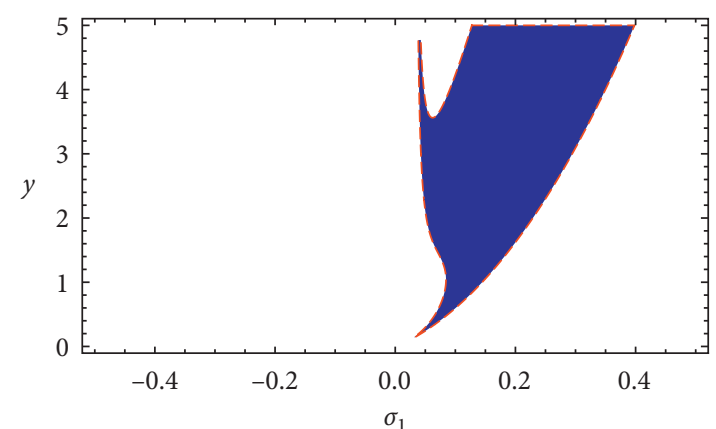

(b)

Figure 8: The system solution behavior in $\gamma-\sigma_{1}$ plane when $y_{0}=0.07$ : (a) the controlled system without time delay; (b) the controlled system with $\tau=\tau_{1}+\tau_{2}=0.03$.

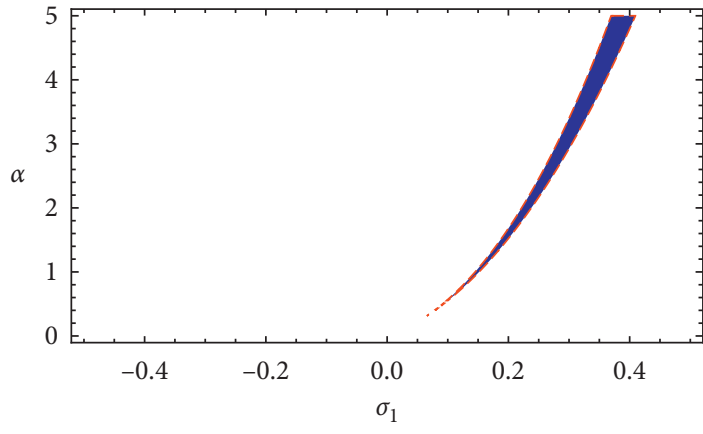

(a)

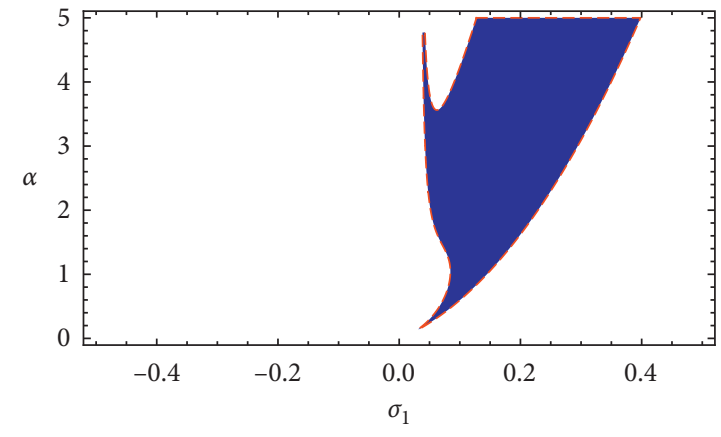

(b)

Figure 9: The system solution behavior in $\alpha-\sigma_{1}$ plane when $y_{0}=0.07$ : (a) the controlled system without time delay; (b) the controlled system with $\tau=\tau_{1}+\tau_{2}=0.03$.

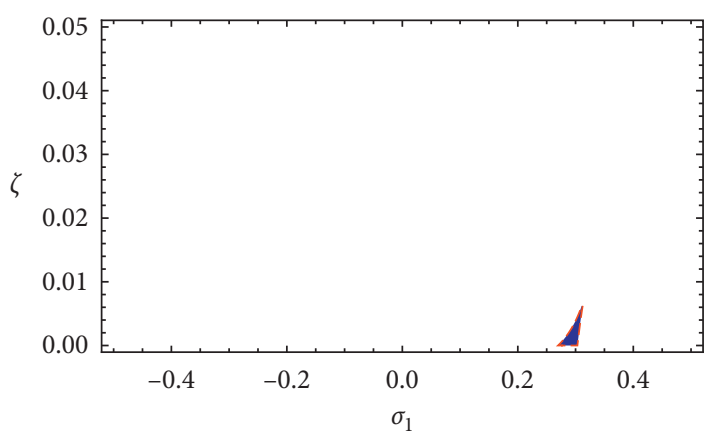

(a)

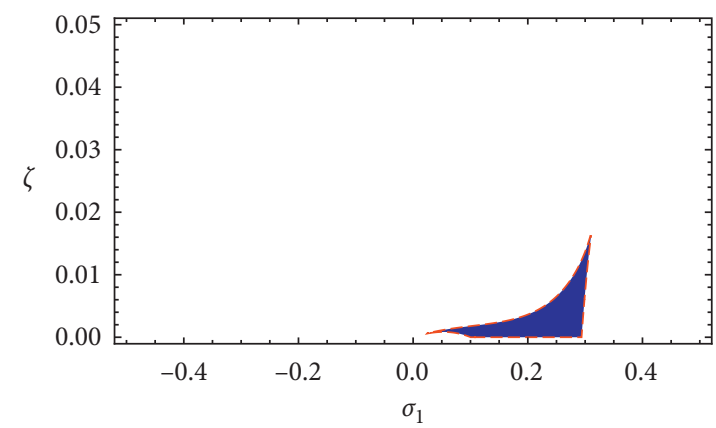

(b)

Figure 10: The system solution behavior in $\zeta-\sigma_{1}$ plane when $y_{0}=0.07$ : (a) the controlled system without time delay; (b) the controlled system with $\tau=\tau_{1}+\tau_{2}=0.03$.

$$
\left\{\begin{array}{l}
\frac{\mathrm{d} A_{1}}{\mathrm{~d} \Omega}=-\frac{\left(\mathrm{d} m_{3} / \mathrm{d} \Omega\right) A_{1}^{3}+\left(\mathrm{d} m_{2} / \mathrm{d} \Omega\right) A_{1}^{2}+\left(\mathrm{d} m_{1} / \mathrm{d} \Omega\right) A_{1}+\left(\mathrm{d} m_{0} / \mathrm{d} \Omega\right)}{3 m_{3} A_{1}^{2}+2 m_{2} A_{1}+m_{1}}=0, \\
m_{3} A_{1}^{3}+m_{2} A_{1}^{2}+m_{1} A_{1}+m_{0}=0,
\end{array}\right.
$$




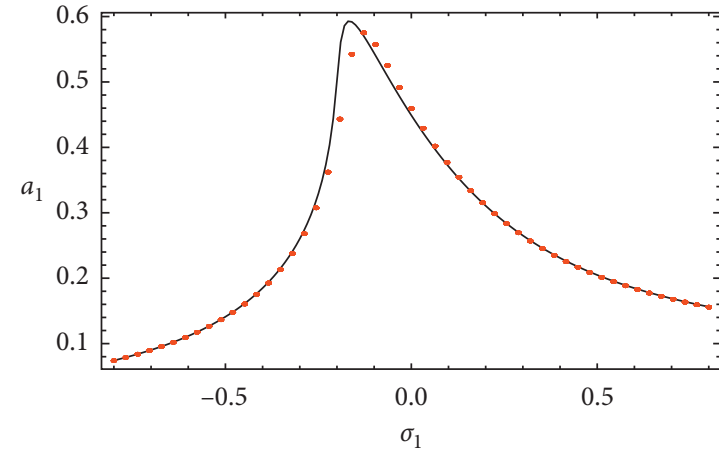

(a)

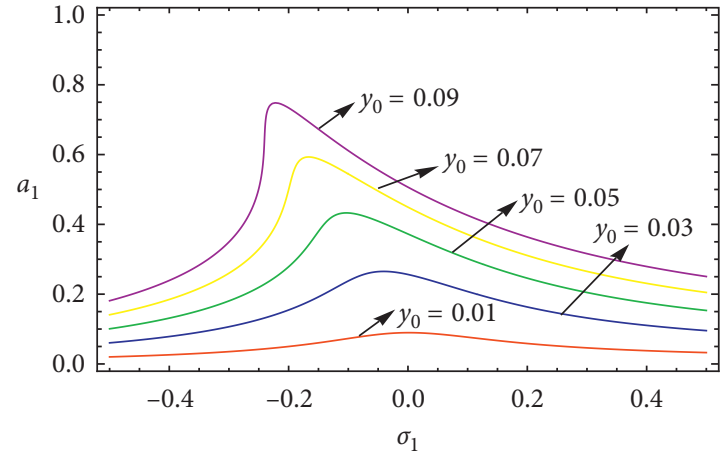

(b)

Figure 11: Response curves of the uncontrolled beam. (a) Comparison between numerical solutions and approximate solutions when $y_{0}=0.07$; (b) effect of $y_{0}$ on the response curves.

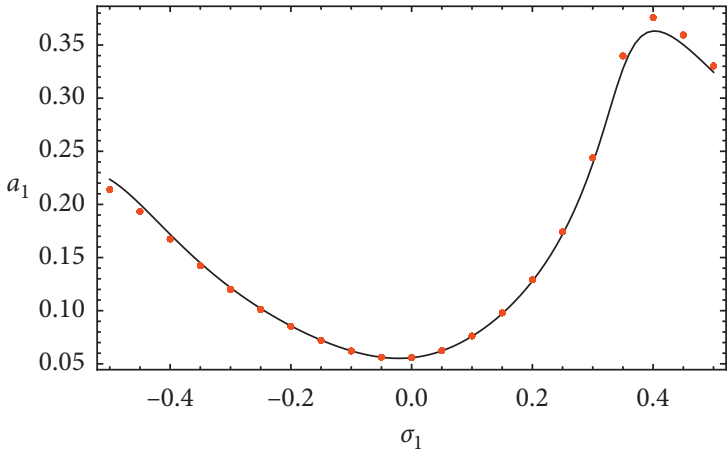

(a)

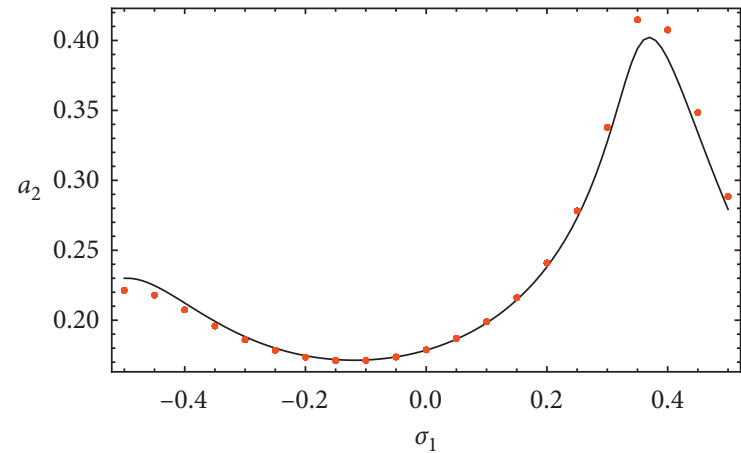

(b)

FiguRE 12: Frequency $\sigma_{1}$-response curves for the controlled system with $\tau_{1}=0.02, \tau_{2}=0.01, \alpha=3, \gamma=3$, $\zeta=0.05$, and $y_{0}=0.07$ : (a) the main system; (b) the controller.

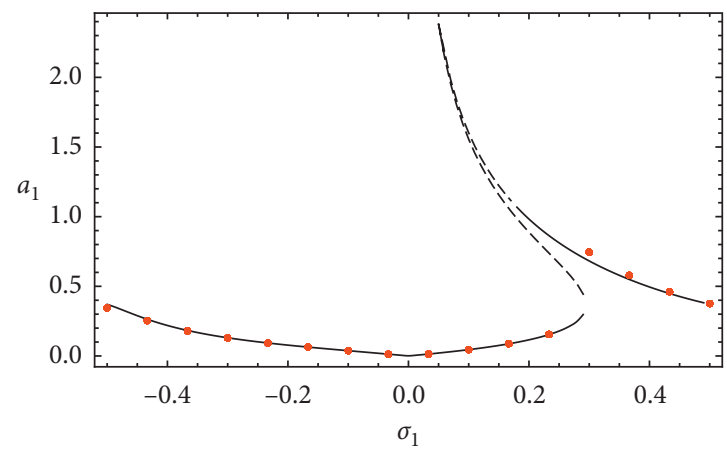

(a)

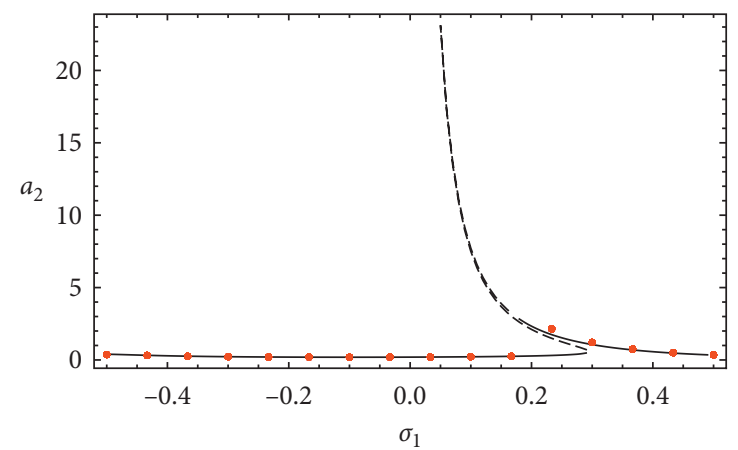

(b)

FIGURE 13: Frequency $\sigma_{1}$-response curves for the controlled system with $\tau_{1}=0.02, \tau_{2}=0.01, \alpha=3, \gamma=3, \zeta=0.001$, and $y_{0}=0.07$ : (a) the main system; (b) the controller.

and the following inequality:

$$
\frac{\mathrm{d}^{2} A_{1}}{\mathrm{~d} \Omega^{2}}<0
$$

Based on equations (49) and (50), we give the excitation frequency $\Omega$ which minimizes the amplitude of the main system when the natural frequency of the controller $\omega_{2}$ varies for two different cases in Tables 1 and 2. Table 1 shows 


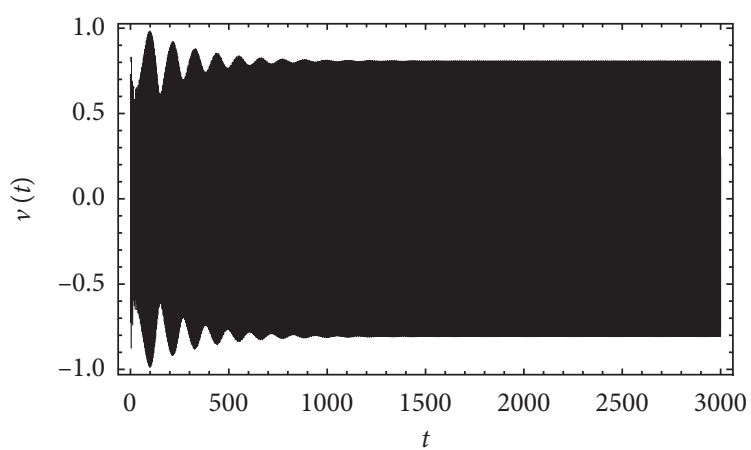

(a)

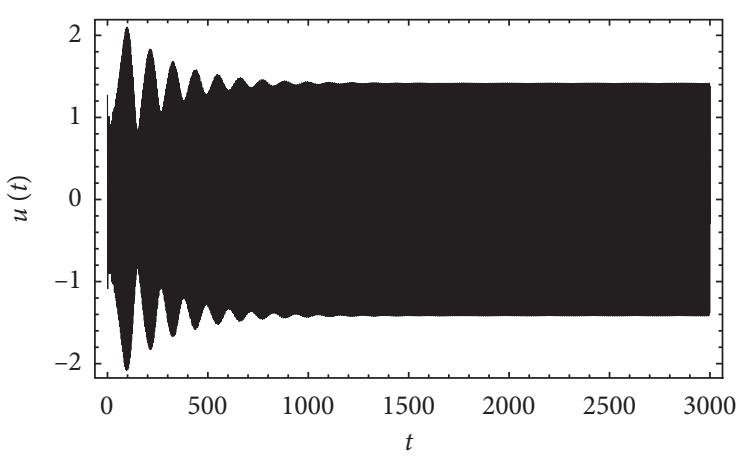

(b)

FIgURE 14: Time history for the controlled system when $\tau_{1}=0.02, \tau_{2}=0.01, \sigma_{1}=0.28, y_{0}=0.07$ with the initial condition $v(0)=0.748, u(0)=1.27, \dot{v}(0)=0.1$, and $\dot{u}(0)=0.01$ : (a) the main system; (b) the controller.

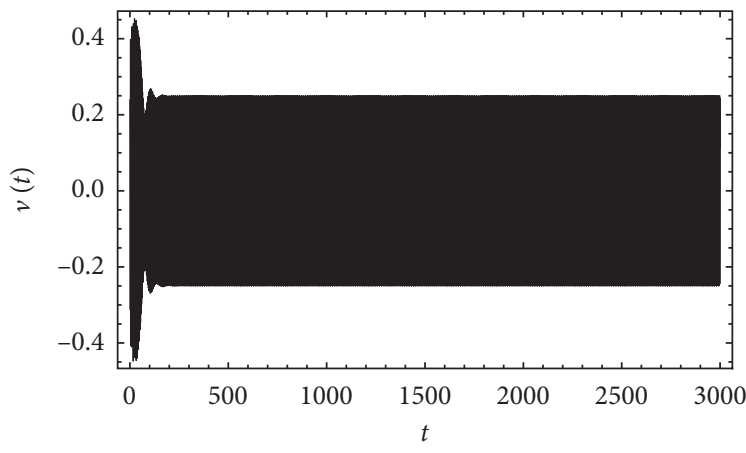

(a)

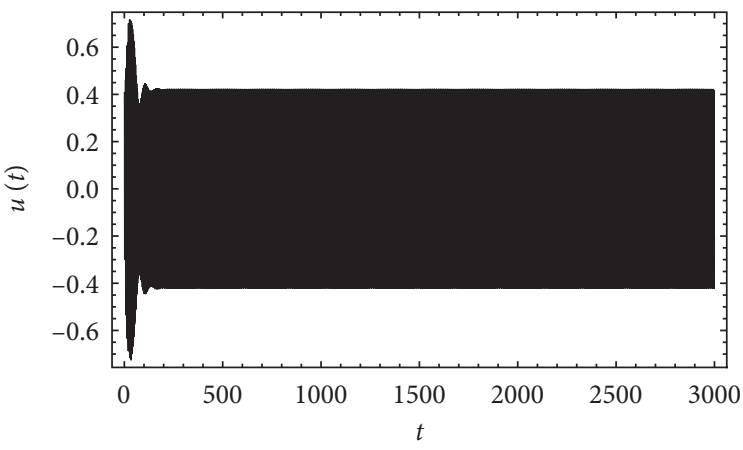

(b)

FIGURE 15: Time history for the controlled system when $\tau_{1}=0.02, \tau_{2}=0.01, \sigma_{1}=0.28, y_{0}=0.07$ with the initial condition $v(0)=0.239, u(0)=0.408, \dot{v}(0)=0.1$, and $\dot{u}(0)=0.01$ : (a) the main system; (b) the controller.

TABle 1: Different minimum points of amplitude $a_{1}$ when $\omega_{1}=3.06, \zeta=0.05, y_{0}=0.07$, and $\tau=\tau_{1}+\tau_{2}=0.05$.

\begin{tabular}{lccc}
\hline$\omega_{2}$ & $\Omega$ & $A_{1}$ & $a_{1}$ \\
\hline 2.56 & 2.56087 & $7.41 \times 10^{-4}$ & 0.02722 \\
2.66 & 2.65812 & $1.01 \times 10^{-3}$ & 0.03172 \\
2.76 & 2.7545 & $1.352 \times 10^{-3}$ & 0.03677 \\
2.86 & 2.84991 & $1.796 \times 10^{-3}$ & 0.04237 \\
2.96 & 2.9442 & $2.353 \times 10^{-3}$ & 0.04851 \\
3.06 & 3.03739 & $3.0360 \times 10^{-3}$ & 0.055099 \\
3.16 & 3.12935 & $3.8429 \times 10^{-3}$ & 0.061991 \\
3.26 & 3.22017 & $4.755 \times 10^{-3}$ & 0.068954 \\
3.36 & 3.30998 & $5.7294 \times 10^{-3}$ & 0.075693 \\
3.46 & 3.39906 & $6.7057 \times 10^{-3}$ & 0.081882 \\
3.56 & 3.48771 & $7.6153 \times 10^{-3}$ & 0.08727 \\
\hline
\end{tabular}

that the excitation frequency $\Omega$ and the controller natural frequency $\omega_{2}$ that minimize the amplitude of the main system always deviate from each other when $\zeta=0.05, \tau_{1}+\tau_{2}=0.05$. And with the increase of $\sigma_{2}$ (i.e. $\left.\omega_{2}-\omega_{1}\right)$, the deviation $\left|\Omega-\omega_{2}\right|$ becomes bigger and bigger. For example, for $\sigma_{2}=0.5$ the minimum steady-state amplitude of the main system occurs at $\sigma_{1}=0.43$. Table 2 shows that the excitation frequency $\Omega$ and the controller natural
TABLe 2: Different minimum points of amplitude $a_{1}$ when $\omega_{1}=3.06, \zeta=0.001, y_{0}=0.07$, and $\tau=\tau_{1}+\tau_{2}=0.05$.

\begin{tabular}{lccc}
\hline$\omega_{2}$ & $\Omega$ & $A_{1}$ & $a_{1}$ \\
\hline 2.56 & 2.56 & $3.58 \times 10^{-7}$ & $5.98 \times 10^{-4}$ \\
2.66 & 2.66 & $4.86 \times 10^{-7}$ & $6.98 \times 10^{-4}$ \\
2.76 & 2.76 & $6.53 \times 10^{-7}$ & $8.08 \times 10^{-4}$ \\
2.86 & 2.86 & $8.68 \times 10^{-7}$ & $9.31 \times 10^{-4}$ \\
2.96 & 2.95999 & $1.14 \times 10^{-6}$ & $1.07 \times 10^{-3}$ \\
3.06 & 3.05999 & $1.49 \times 10^{-6}$ & $1.22 \times 10^{-3}$ \\
3.16 & 3.15999 & $1.93 \times 10^{-6}$ & $1.39 \times 10^{-3}$ \\
3.26 & 3.25998 & $2.47 \times 10^{-6}$ & $1.57 \times 10^{-3}$ \\
3.36 & 3.35997 & $3.15 \times 10^{-6}$ & $1.77 \times 10^{-3}$ \\
3.46 & 3.45996 & $3.98 \times 10^{-6}$ & $1.99 \times 10^{-3}$ \\
3.56 & 3.55995 & $5.00 \times 10^{-6}$ & $2.24 \times 10^{-3}$ \\
\hline
\end{tabular}

frequency $\omega_{2}$ that minimize the amplitude of the main system are almost equal to each other when $\zeta=0.001, \tau_{1}+\tau_{2}=0.05$. It can be seen that the minimum amplitudes of the main system are almost zero when $\zeta=0.001$. From these tables, we conclude that it is necessary to tune the controller's natural frequency $\omega_{2}$ to the same value of the excitation frequency $\Omega$ only when the controller damping coefficient $\zeta$ is close to zero. Figure 16 shows time 


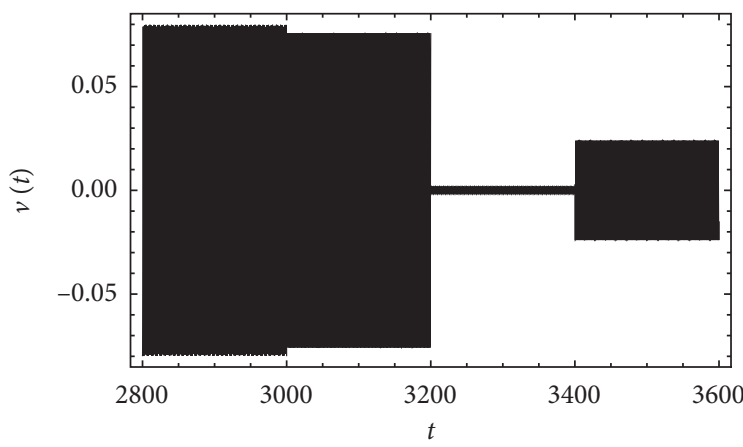

(a)

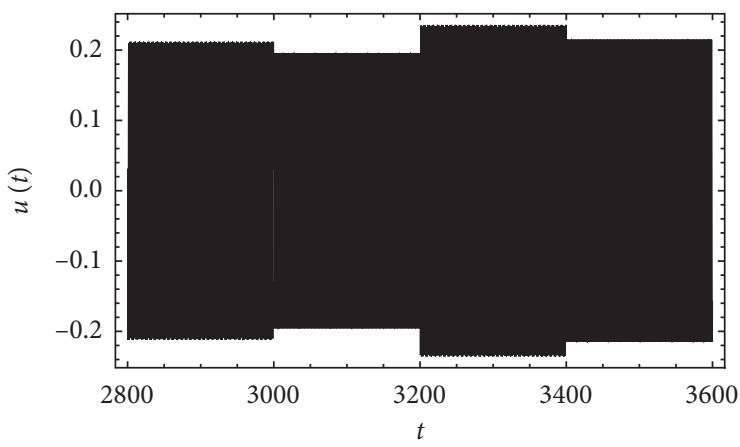

(b)

FIgURE 16: Time history for the controlled system when $\tau_{1}=0.03, \tau_{2}=0.02, y_{0}=0.07: 2800 \leq t \leq 3000, \zeta=0.05, \Omega=\omega_{2}=3.36$; $3000 \leq t \leq 3200, \zeta=0.05, \Omega=3.31, \omega_{2}=3.36 ; 3200 \leq t \leq 3400, \zeta=0.001, \Omega=\omega_{2}=3.36$; and $3400 \leq t \leq 3600, \zeta=0.001, \Omega=3.31, \omega_{2}=3.36$ : (a) the main system; (b) the controller.

histories of the primary system and the controller for four different cases, i.e., $\zeta=0.05, \Omega=\omega_{2}=3.36$; $\zeta=0.05, \Omega=3.31, \omega_{2}=3.36 ; \zeta=0.001, \Omega=\omega_{2}=3.36$ and $\zeta=0.001, \Omega=3.31, \omega_{2}=3.36$. From Figure $16(\mathrm{a})$, it is observed that the amplitude of the main system when $\zeta=$ $0.05, \Omega=3.31, \omega_{2}=3.36$ is smaller than that when $\zeta=0.05, \Omega=\omega_{2}=3.36$. It can be seen that the best condition for vibration reduction in the four cases is that $\zeta=$ 0.001 and $\Omega=\omega_{2}$. From Figure 16, we also observe that decreasing the controller damping coefficient $\zeta$ can better suppress the vibration of the main system. However, it can enhance the vibration of the controller.

In general, only when the controller damping coefficient $\zeta$ approaches zero will the minimum main system amplitude occur at $\sigma_{1}=\sigma_{2}$. And in this case, the vibration of the main system almost vanishes.

\section{Conclusion}

In this paper, a time-delayed positive position feedback (PPF) controller has been investigated for the simultaneously resonance case $\left(\Omega=\omega_{1}, \omega_{1}=\omega_{2}\right)$ of a dynamical system. The integral iterative method is used to derive the second-order approximations and the amplitude equations. Then, the stability of the periodic solution is studied by utilizing a combination of Floquet theory and Hill's determinant. The analysis revealed the following:

(1) The analytical results obtained by the integral iterative method correspond well with numerical simulations both quantitatively and qualitatively.

(2) The amplitude or stability of the controlled system (including the main system and the controller) does not depend on the individual time delay $\tau_{1}, \tau_{2}$, but on their summation $\tau_{1}+\tau_{2}$.

(3) The unavoidable total time delay $\tau_{1}+\tau_{2}$ in the control loop should not exceed 0.08 . Otherwise, the controlled system may destabilize.

(4) Although the uncontrolled beam is nonlinear in form, it does not show nonlinear characteristics when $y_{0}$ varies in $(0,0.1)$.
(5) The appearance of time delay and near zero damping coefficient $\zeta$ are two main factors to enhance the nonlinear characteristics of the controlled system. We can adjust different initial values to achieve the effect of vibration reduction in the region where multiple solutions exist.

(6) Only when the controller damping coefficient $\zeta$ approaches zero will the minimum amplitude of the main system occur at $\Omega=\omega_{2}$. That is to say, if the controller damping coefficient $\zeta$ is very close to zero, device should be designed to trace the excitation frequency and modifies the controller natural frequency adaptively to the same new value of the excitation frequency.

\section{Comparison with Previously Published Work}

In recent years, the vibration control for flexible beam structure has received much attention $[2-5,21]$. First of all, the approximate solutions of the considered system in the above works were obtained by applying the multiple time scales perturbation technique and the stability of the steadystate solution is ascertained by using frequency-response equations. In [2], Li employed a PPF controller to reduce the high-amplitude vibration of the single mode of the flexible beam when subjected to primary resonance excitation. The author concluded that the PPF control scheme possessed a wide suppression bandwidth if the absorber's frequency is properly tuned. Warminski et al. [3] used four different controllers to control the high-amplitude vibration of a flexible, geometrically nonlinear beam and showed that the positive position feedback controller and the nonlinear saturation controller were most effective. However, they only presented an analytical study for the NSC controlled system. Mitura et al. [4] utilized the PPF algorithm for vibration reduction of a horizontal beam near the primary resonance and a vertical beam near principal parametric resonance from a numerical point of view. The results showed that the vibration suppression effect of vertical beam was much better than that of horizontal beam. The PPF control method for the horizontal beam needed further 
improvement. In $[5,16]$, the authors investigated positive position feedback controllers for suppression of nonlinear beam vibration. They graphically inferred that the minimum amplitude of the main system occurred when the excitation frequency equaled the controller natural frequency.

Based on the PPF controlled system in [4], we consider the effect of time delays on the controller performance in this paper. The approximate solutions are obtained by the integral iterative method and the stability of the steady-state solution is studied by utilizing a combination of Floquet theory and Hill's determinant. The effects of various control parameters on time delay stability margins and the existence of multiple-solution region are investigated. The location of the minimum steady state main system amplitude is studied analytically. We found that only when the controller damping coefficient approaches zero will the minimum main system amplitude occur when the excitation frequency equaled the controller natural frequency.

\section{Appendix}

\section{The Integral Iterative Method}

Vibrations of various kinds with time delay can be governed by delay differential equations of the form

$$
\begin{aligned}
\ddot{x}_{i}+\lambda_{i} x_{i} & =\phi_{i}\left(x_{i}, \dot{x}_{i}, x_{i \tau}, \dot{x}_{i \tau}, x_{j}, \dot{x}_{j}, x_{j \tau}, \dot{x}_{j \tau} ; \varepsilon_{p_{i}}, t\right) \\
& =\phi_{i}[t, \tau], i, j=1,2, \ldots,
\end{aligned}
$$

where $x_{i \tau}=x_{i}(t-\tau)$ and $\varepsilon_{p_{i}}$ is a parameter.

Theorem 1. Every periodic solution of the differential equation (A.1) is a solution of the integrodifferential equation (see $[18-20,24])$

$$
x_{i}(t)=\int_{0}^{2 \pi} G_{i}[t, \sigma] \phi_{i}[\sigma] \mathrm{d} \sigma+\delta_{\lambda_{i}}^{n_{i}^{2}}\left(r_{i} \cos n_{i} t+s_{i} \sin n_{i} t\right),
$$

and the time delay terms can be transformed into

$$
\begin{aligned}
x_{i}(t-\tau)= & \int_{0}^{2 \pi} G_{i}[t-\tau, \sigma] \phi_{i}[\sigma] \mathrm{d} \sigma+\delta_{\lambda_{i}}^{n_{i}^{2}}\left(r_{i} \cos n_{i}(t-\tau)\right. \\
& \left.+s_{i} \sin n_{i}(t-\tau)\right),
\end{aligned}
$$

where

$G_{i}[t, \sigma]=(1 / \pi)\left(\left(1 / 2 \lambda_{i}\right)+\sum_{j=1}^{\infty}\left((\cos j(t-\sigma)) / \vartheta \lambda_{i}-j^{2}\right)\right)$, $G_{i}[t-\tau, \sigma]=1 / \pi\left(\left(1 / 2 \lambda_{i}\right)+\sum_{j=1}^{\infty}(\cos j \tau \cos j(t-\sigma)+\right.$

$\sin j \tau \sin j(t-\sigma)) /\left(\vartheta \lambda_{i}-j^{2}\right)$ are the corresponding generalized Green's function, where

$$
\delta_{\lambda_{i}}^{n^{2}}= \begin{cases}1, & \lambda_{i}=n_{i}^{2}, n_{i} \text { being an integer, } \\ 0, & \text { otherwise, }\end{cases}
$$

is the Kronecker symbol and $\vartheta=\vartheta_{\lambda_{i}}^{n_{i}^{2}}=1-\delta_{\lambda_{i}}^{n_{i}^{2}}$.

If $\lambda_{i}=n_{i}^{2}$ ( $n_{i}$ being an integer for the resonance case), the parameters $r_{i}, s_{i}$ can be determined by the periodicity equations (see [19])

$$
\begin{aligned}
& r_{i}=\frac{1}{\pi} \int_{0}^{2 \pi} x_{i}(t) \cos n_{i} t \mathrm{~d} t, \\
& s_{i}=\frac{1}{\pi} \int_{0}^{2 \pi} x_{i}(t) \sin n_{i} t \mathrm{~d} t,
\end{aligned}
$$

which are equivalent to

$$
\int_{0}^{2 \pi} \phi_{i}[t] \cos n_{i} t \mathrm{~d} t=\int_{0}^{2 \pi} \phi_{i}[t] \sin n_{i} t \mathrm{~d} t=0 .
$$

The solutions of (A.2) and (A.3) can be found by successive approximations of $x_{i k}(t), x_{i k}(t-\tau), k=1,2,3, \ldots$, which are given by

$$
\begin{aligned}
& x_{i k}(t)=\int_{0}^{2 \pi} G_{i}[t, \sigma] \phi_{i, k-1}[\sigma, \tau] \mathrm{d} \sigma+\delta_{\lambda_{i}}^{n_{i}^{2}}\left(r_{i} \cos \left(n_{i} t\right)\right. \\
& \left.+s_{i} \sin \left(n_{i} t\right)\right), \\
& \quad x_{i k}(t-\tau)=\int_{0}^{2 \pi} G_{i}[t-\tau, \sigma] \phi_{i, k-1}[\sigma, \tau] \mathrm{d} \sigma \\
& \quad+\delta_{\lambda_{i}}^{n_{i}^{2}}\left(r_{i} \cos n_{i}(t-\tau)+s_{i} \sin n_{i}(t-\tau)\right),
\end{aligned}
$$

where

$$
\begin{aligned}
& \phi_{i 0}[t, \tau]= \begin{cases}0, & \lambda_{i}=n_{i}^{2}, \\
\phi\left[0,0,0,0,0,0,0,0 ; \varepsilon_{p_{i}}, t\right], & \lambda_{i} \neq n_{i}^{2},\end{cases} \\
& \phi_{i k}[t, \tau]=\phi_{i}\left[x_{i k}(t), \dot{x}_{i k}(t), x_{i k}(t-\tau), \dot{x}_{i k}(t-\tau), x_{j k}(t)\right. \text {, } \\
& \left.\dot{x}_{j k}(t), x_{j k}(t-\tau), \dot{x}_{j k}(t-\tau) ; \varepsilon_{p}, t\right] \text {, } \\
& k=1,2,3, \ldots \text {. }
\end{aligned}
$$

\section{Data Availability}

The data used to support the findings of this study are included within the article.

\section{Conflicts of Interest}

The authors declare that they have no conflicts of interest.

\section{Acknowledgments}

This work was supported by the Youth Program of National Natural Science Foundation of China under Grant No. 11702250 and National Natural Science Foundation of China under Grant Nos. 11872185, 11972327, and 11771407.

\section{References}

[1] J. Shan, H. Liu, and D. Sun, "Slewing and vibration control of a single-link flexible manipulator by positive position feedback (PPF)," Mechatronics, vol. 15, no. 3, pp. 487-503, 2005.

[2] L. Jun, "Positive position feedback control for high-amplitude vibration of a flexible beam to a principal resonance excitation," Shock and Vibration, vol. 17, no. 2, pp. 187-203, 2010.

[3] J. Warminski, M. Bochenski, W. Jarzyna, P. Filipek, and M. Augustyniak, "Active suppression of nonlinear composite 
beam vibrations by selected control algorithms," Communications in Nonlinear Science and Numerical Simulation, vol. 16, no. 5, pp. 2237-2248, 2011.

[4] A. Mitura, J. Warminski, and M. Bochenski, "Optimal control methods for vertical and horizontal beam dynamics," Journal of Physics: Conference Series, vol. 382, Article ID 012041, 2012.

[5] W. A. El-Ganaini, N. A. Saeed, and M. Eissa, "Positive position feedback (PPF) controller for suppression of nonlinear system vibration," Nonlinear Dynamics, vol. 72, no. 3, pp. 517-537, 2013.

[6] E. Omidi and S. N. Mahmoodi, "Nonlinear vibration suppression of flexible structures using nonlinear modified positive position feedback approach," Nonlinear Dynamics, vol. 79, no. 2, pp. 835-849, 2015.

[7] A. T. El-sayed and H. S. Bauomy, "Nonlinear analysis of vertical conveyor with positive position feedback (PPF) controllers," Nonlinear Dynamics, vol. 83, no. 1-2, pp. 919939, 2016.

[8] A. Kandil and M. Eissa, "Improvement of positive position feedback controller for suppressing compressor blade oscillations," Nonlinear Dynamics, vol. 90, no. 3, pp. 1727-1753, 2017.

[9] N. A. Saeed and M. Kamel, “Active magnetic bearing-based tuned controller to suppress lateral vibrations of a nonlinear Jeffcott rotor system," Nonlinear Dynamics, vol. 90, no. 1, pp. 457-478, 2017.

[10] M. Eissa and N. A. Saeed, "Nonlinear vibration control of a horizontally supported Jeffcott-rotor system," Journal of Vibration and Control, vol. 24, no. 24, pp. 5898-5921, 2018.

[11] N. A. Saeed and H. A. El-Gohary, "Influences of time-delays on the performance of a controller based on the saturation phenomenon," European Journal of Mechanics-A/Solids, vol. 66, pp. 125-142, 2017.

[12] N. A. Saeed and W. A. El-Ganaini, "Utilizing time-delays to quench the nonlinear vibrations of a two-degree-of-freedom system," Meccanica, vol. 52, no. 11-12, pp. 2969-2990, 2017.

[13] N. A. Saeed and W. A. El-Ganaini, "Time-delayed control to suppress the nonlinear vibrations of a horizontally suspended Jeffcott-rotor system," Applied Mathematical Modelling, vol. 44, pp. 523-539, 2017.

[14] N. A. Saeed, W. A. El-Ganaini, and M. Eissa, "Nonlinear time delay saturation-based controller for suppression of nonlinear beam vibrations," Applied Mathematical Modelling, vol. 37, no. 20-21, pp. 8846-8864, 2013.

[15] W. A. El-Ganaini, A. Kandil, M. Eissa, and M. Kamel, "Effects of delayed time active controller on the vibration of a nonlinear magnetic levitation system to multi excitations," Journal of Vibration and Control, vol. 22, no. 5, pp. 1257-1275, 2016.

[16] H. Abdelhafez and M. Nassar, "Effects of time delay on an active vibration control of a forced and self-excited nonlinear beam," Nonlinear Dynamics, vol. 86, no. 1, pp. 137-151, 2016.

[17] A. Kandil and W. A. El-Ganaini, "Investigation of the time delay effect on the control of rotating blade vibrations," European Journal of Mechanics-A/Solids, vol. 72, pp. 16-40, 2018.

[18] J. Xu, Y. Chen, and K. W. Chung, "An improved time-delay saturation controller for suppression of nonlinear beam vibration," Nonlinear Dynamics, vol. 82, no. 4, pp. 1691-1707, 2015.

[19] G. Schmidt and A. Tondl, Nonlinear Vibrations, Cambridge University Press, Cambridge, UK, 1986.
[20] Y. Chen and J. Xu, "Applications of the integral equation method to delay differential equations," Nonlinear Dynamics, vol. 73, no. 4, pp. 2241-2260, 2013.

[21] J. Warminski, M. P. Cartmell, A. Mitura, and M. Bochenski, "Active vibration control of a nonlinear beam with self- and external excitations," Shock and Vibration, vol. 20, no. 6, pp. 1033-1047, 2013.

[22] A. H. Nayfeh, C.-M. Chin, and J. Pratt, "Perturbation methods in nonlinear dynamics-applications to machining dynamics," Journal of Manufacturing Science and Engineering, vol. 119, no. 4A, pp. 485-493, 1997.

[23] M. F. Daqaq, K. A. Alhazza, and H. N. Arafat, "Non-linear vibrations of cantilever beams with feedback delays," International Journal of Non-linear Mechanics, vol. 43, no. 9, pp. 962-978, 2008.

[24] Y. Chen, K.-W. Chung, J. Xu, and Y. Sun, "Analysis of vibration suppression of master structure in nonlinear systems using nonlinear delayed absorber," International Journal of Dynamics and Control, vol. 2, no. 1, pp. 55-67, 2014. 